\title{
Global Connectivity and Function of Descending Spinal Input Revealed by 3D Microscopy and Retrograde Transduction
}

\author{
Zimei Wang, ${ }^{1 \star}$ Brian Maunze, ${ }^{1 \star}$ Yunfang Wang, ${ }^{2}$-Pantelis Tsoulfas, ${ }^{2}$ and Murray G. Blackmore ${ }^{1}$ \\ ${ }^{1}$ Department of Biomedical Sciences, Marquette University, Milwaukee, Wisconsin 53211, and 2Department of Neurological Surgery, Miami Project to Cure \\ Paralysis, University of Miami Miller School of Medicine, Miami, Florida 33136
}

The brain communicates with the spinal cord through numerous axon tracts that arise from discrete nuclei, transmit distinct functions, and often collateralize to facilitate the coordination of descending commands. This complexity presents a major challenge to interpreting functional outcomes from therapies that target supraspinal connectivity after injury or disease, while the wide distribution of supraspinal nuclei complicates the delivery of therapeutics. Here we harness retrograde viral vectors to overcome these challenges. We demonstrate that injection of AAV2-Retro to the cervical spinal cord of adult female mice results in highly efficient transduction of supraspinal populations throughout the brainstem, midbrain, and cortex. Some supraspinal populations, including corticospinal and rubrospinal neurons, were transduced with $>90 \%$ efficiency, with robust transgene expression within $3 \mathrm{~d}$ of injection. In contrast, propriospinal and raphe spinal neurons showed much lower rates of retrograde transduction. Using tissue clearing and light-sheet microscopy we present detailed visualizations of descending axons tracts and create a mesoscopic projectome for the spinal cord. Moreover, chemogenetic silencing of supraspinal neurons with retrograde vectors resulted in complete and reversible forelimb paralysis, illustrating effective modulation of supraspinal function. Retrograde vectors were also highly efficient when injected after spinal injury, highlighting therapeutic potential. These data provide a global view of supraspinal connectivity and illustrate the potential of retrograde vectors to parse the functional contributions of supraspinal inputs.

Key words: corticospinal; DREADD; light-sheet microscopy; motor control; retrograde gene therapy; supraspinal centers

Significance Statement

The complexity of descending inputs to the spinal cord presents a major challenge in efforts deliver therapeutics to widespread supraspinal systems, and to interpret their functional effects. Here we demonstrate highly effective gene delivery to diverse supraspinal nuclei using a retrograde viral approach and combine it with tissue clearing and 3D microscopy to map the descending projectome from brain to spinal cord. These data highlight newly developed retrograde viruses as therapeutic and research tools, while offering new insights into supraspinal connectivity.

\section{Introduction}

Descending axon tracts in the spinal cord originate from diverse nuclei throughout forebrain, midbrain, and hindbrain, and serve

\footnotetext{
Received May 11, 2018; revised 0ct. 3, 2018; accepted 0ct. 9, 2018.

Author contributions: B.M. wrote the first draft of the paper; P.T. edited the paper; Z.W., P.T., and M.G.B. designed research; Z.W., B.M., Y.W., and M.G.B. performed research; B.M., P.T., and M.G.B. analyzed data; M.G.B. wrote the paper.

This work was supported by Grants from NINDS, the Bryon Riesch Paralysis Foundation, The Miami Project to Cure Paralysis, and the Buoniconti Fund.

The authors declare no competing financial interests.

*Z.W. and B.M. contributed equally to this work.

Correspondence should be addressed to either of the following: Murray G. Blackmore, Marquette University, Schroeder Complex 446, 561 N 16th St., Milwaukee, WI 53211, E-mail: murray.blackmore@marquette.edu; or Pantelis Tsoulfas, University of Miami, The Miami Project to Cure Paralysis 1095 NW 14th Terrace (R-48) Miami, FL 33136, E-mail: ptsoulfa@med.miami.edu.
}

a wide range of motor, sensory, and autonomic functions (Liang et al., 2011; Kim et al., 2017). Coordinated activity in multiple tracts often converges on the same motor output. For example, distinct phases of targeted forelimb movement are controlled by different sets of corticospinal tract distributed through motor cortex, in coordination with other nuclei including the red nucleus and reticular formation (Kuypers and Martin, 1982; Alstermark et al., 2004; Lemon, 2008; Esposito et al., 2014; Siegel et al., 2015; Wang et al., 2017). Clarifying the different contributions of supraspinal populations to basic motor control remains a challenge. In addition, this complexity presents a significant chal-

https://doi.org/10.1523/JNEUROSCl.1196-18.2018

Copyright $\odot 2018$ the authors $\quad 0270-6474 / 18 / 3810566-16 \$ 15.00 / 0$ 
lenge for the study and the treatment of spinal injuries. Spinal injuries in both human patients and animal models are highly variable, leaving each individual with different subsets of descending axon tracts intact. Thus, a major question in the field is how plasticity in distinct supraspinal tracts, both spontaneous and treatment-induced, yields behavioral improvements. Conversely, the fact that supraspinal neurons are distributed so widely and carry both discrete and overlapping functions presents a challenge for the delivery of therapeutics. Genetic interventions that boost the intrinsic growth ability of supraspinal neurons are showing promise in enhance regenerative axon growth from various types of neurons including corticospinal, rubrospinal, and reticulospinal projections (Liu et al., 2010, 2017; Blackmore et al., 2012; Wang et al., 2015; Geoffroy et al., 2016; $\mathrm{Wu}$ et al., 2017). In these proof-of-principle studies vectors are typically delivered by direct brain injection, yet this approach is impractical to reach all supraspinal neurons in rodent models, let alone human patients.

The development of new viral vectors with effective retrograde transduction properties affords new opportunities to address these challenges. Descending axons converge to relatively small target fields in the spinal cord, and thus a small number of retrograde injections could potentially affect widely distributed supraspinal neurons of origin (Frampton et al., 2005; Nassi et al., 2015). The potential utility of this retrograde approach is well recognized, but has been limited by inadequate efficiency of transduction, particularly for corticospinal tract neurons (Jara et al., 2012; Klaw et al., 2013). Here we harness a recently developed adeno-associated serotype termed AAV2-Retro as a tool to clarify supraspinal connectivity and function (Tervo et al., 2016). We first demonstrate that AAV2-Retro delivered to the cervical and lumbar spinal cord drives rapid and highly efficient transduction of supraspinal populations including reticular, red nucleus, and corticospinal. Importantly, retrograde transduction remained equally effective when vectors were delivered after spinal injury, highlighting the potential for therapeutic delivery. We then use tissue clearing and light-sheet microscopy to present detailed visualizations of supraspinal neurons and descending axon tracts in whole brain preparations. Finally, we test a retrograde viral approach to probe the function of supraspinal inputs. We found that after retrograde delivery of inhibitory designer receptors exclusively activated by designer drugs (DREADDs), receptor activation in transduced supraspinal populations was sufficient to induce complete forelimb paralysis. Moreover, when we used transgenic targeting in conjunction with Retro-DREADD delivery to enrich expression in corticospinal tract neurons, receptor activation produced movement deficits highly consistent with selective corticospinal tract (CST) ablation. Overall these data highlight the utility of a retrograde viral approach for therapeutic gene delivery, as a tool for neuroanatomical tracing, and for functional dissection of supraspinal circuits.

\section{Materials and Methods}

$A A V$ injection and spinal injuries. All animal procedures were approved by the Marquette University Institutional Animal Care and Use Committee and complied with the National Institutes of Health Guide for the Care and Use of Laboratory Animals. AAV2-Retro-CAG-tdTomato, AAV2-Retro-CAG-EGFP, and AAV2-Retro-CAG-CRE were purchased from the University of North Carolina Viral Vector Core. AAV2-RetrohSyn-DIO-hM4D(Gi)-mCherry (44362) and AAV2-Retro-hSyn-hM4D (Gi)-mCherry (50475) were purchased from Addgene. Adult female C57BL/6 mice ( $>8$ weeks old, $20-22$ g) were anesthetized by ketamine/ xylazine and the cervical spinal column exposed by incision of the skin and blunt dissection of muscles. Mice were mounted in a custom spine stabilizer. AAV particles were mixed with Cholera Toxin Subunit B conjugated to AlexaFluor 488 (CTB-488) in sterile $0.9 \% \mathrm{NaCl}$ (C22841, ThermoFisher, final concentration of $2 \%$ ), and were injected to the spinal cord through a pulled glass micropipette fitted to a $10 \mu \mathrm{l}$ Hamilton syringe driven by a Stoelting QSI pump (catalog \#53311) and guided by a micromanipulator (pumping rate: $0.04 \mu \mathrm{l} / \mathrm{min}$ ). AAV/CTB mixtures were injected between $\mathrm{C} 4$ and $\mathrm{C} 5$ vertebrae, bilaterally, $0.35 \mathrm{~mm}$ lateral to the midline, and to depths of 0.6 and $0.8 \mathrm{~mm}$. For lumbar injections, the T9 vertebrae was used as a landmark to identify the L3 and L4 vertebrae. Injections were made between L3 and L4 vertebrae, bilaterally, $0.35 \mathrm{~mm}$ lateral to the midline, 0.6 and $0.8 \mathrm{~mm}$ depth. Cervical dorsal hemisections were performed as by Blackmore et al. (2012) and Wang et al. (2015). Briefly, mice were anesthetized, their spinal cord exposed, and mounted in a custom spine stabilizer as described above. Using a Vibraknife device (Zhang et al., 2004), in which a rapidly vibrating blade is controlled via a micromanipulator, a transection was made between the fourth and fifth cervical vertebrae, extending from the midline beyond the right lateral edge of the spinal cord, to a depth of $0.85 \mathrm{~mm}$. Injections were performed immediately after injury, unilaterally to right spinal cord, $0.3 \mathrm{~mm}$ rostral to the injury, $0.35 \mathrm{~mm}$ lateral to the midline, and to 0.6 and $0.8 \mathrm{~mm}$ depth.

Tissue processing and imaging. Animals were transcardially perfused with $0.9 \%$ saline and $4 \%$ paraformaldehyde solutions in $1 \times$ PBS $(15710$, Electron Microscopy Sciences). Brains and spinal cords were dissected and fixed overnight in $4 \%$ paraformaldehyde at $4^{\circ} \mathrm{C}$. Spinal cords were embedded in $12 \%$ gelatin in $1 \times$ PBS (G2500, Sigma-Aldrich), and 100 $\mu \mathrm{m}$ transverse sections of the cervical spinal cord cut by vibratome. A complete series of $120 \mu \mathrm{m}$ transverse sections were gathered from each brain. Sections were mounted on slides and imaged with a confocal microscope (Nikon AR1+) using 10× Plan Apochromatic (MRD00105, NA 0.45), $20 \times$ Plan Apochromatic (MRD00205, NA 0,75), or 60× Apochromatic Oil DIC N2 (MRD71600, NA 1.4) objectives. The emitted fluorescence from the sections was strong enough to image without the need to amplify using immunofluorescence methods. The anatomical locations of fluorescent CTB-488 and tdTomato in supraspinal sections were matched to the nomenclature of anatomical structures according to the Allen Brain Atlas. Cells labeled by CTB- 488 and/or tdTomato were manually quantified using the taxonomy tool in NIS-Elements software.

Immunohistochemistry. For 5-HT immunohistochemistry, brainstem sections containing raphespinal neurons were blocked in $10 \%$ goat serum containing $0.04 \%$ Triton- $\mathrm{X}$, and incubated at room temperature for $1 \mathrm{~h}$ while shaking. Brainstem sections were then transferred to a solution ( $3 \%$ goat serum, $0.04 \%$ Triton-X) containing the primary antibody, rabbit anti-5-HT (Sigma-Aldrich, S5545; RRID:AB_477522) at 1:1000 incubated for $18 \mathrm{~h}$ at $4^{\circ} \mathrm{C}$. Sections were then incubated for $2 \mathrm{~h}$ at room temperature in secondary antibody, donkey anti-goat with a fluorescent tag, AlexaFluor 647 (1:500) diluted in (3\% goat serum, 0.04\% Triton-X). To visualize glial fibrillary protein (GFAP), spinal sections were blocked as above and incubated with primary antibody overnight at $4^{\circ}$ (Z0334, DAKO; 1:500; RRID:AB_10013482), rinsed, and then incubated for $2 \mathrm{~h}$ with appropriate AlexaFluor-conjugated secondary antibodies (ThermoFisher; 1:500). Following incubations, sections were mounted on glass slides and coverslipped with mounting medium. Sections were mounted on slides and imaged using a confocal microscope (Nikon AR1+) as above.

DREADD activation and behavioral testing. Clozapine (Sigma-Aldrich, C6305) was prepared as a $1 \mathrm{mg} / \mathrm{ml}$ stock solution in $0.1 \mathrm{M} \mathrm{HCl}$, and then diluted in saline to a final concentration of 10,5 , or $1 \mu \mathrm{g} / \mathrm{ml}$. These solutions were injected intraperitoneally to mice to achieve final doses of $0.01,0.05,0.1$, and $0.2 \mathrm{mg} / \mathrm{kg}$, such that each mouse received between 200 and $400 \mu \mathrm{l}$ total injection (Gomez et al., 2017). Clozapine $N$-oxide (CNO; Tocris Bioscience, 9A/200456) was dissolved in saline to a concentration of $1 \mu \mathrm{g} / \mathrm{ml}$ and injected intraperitoneally to a final dose of 10 $\mathrm{mg} / \mathrm{kg}$. Immediately after injection mice were placed in a clear-bottomed cage and digitally recorded from below. At 0, 5, 10, $15 \mathrm{~min}$, and then hourly thereafter, animal forelimb movement was scored on the following scale: $5=$ normal locomotion with predominantly plantar placement, 4 = weight-bearing locomotion but with frequent non-plantar placement (curled forelimb placed on ventral surface), $3=$ weight- 
Table 1. Overview of experimental groups, sample sizes, and variables

\begin{tabular}{|c|c|c|c|c|}
\hline Experiment & Total mice & Survival time & Virus treatment and titer & Imaging \\
\hline CTB colabel (uninjured) & 13 & 3,7, or $14 \mathrm{~d}$ & $\begin{array}{l}\text { Retro-AAV-tdTomato }\left(3.8 \times 10^{12} \mathrm{GC} / \mathrm{ml}\right) \\
\text { Retro-AAV-EGFP }\left(3.9 \times 10^{12} \mathrm{GC} / \mathrm{ml}\right)\end{array}$ & $2 D$ \\
\hline CTB colabel (injured) & 8 & $28 d$ & Retro-AAV-tdTomato $\left(3.8 \times 10^{12} \mathrm{GC} / \mathrm{ml}\right)$ & $2 D$ \\
\hline Cervical retrograde (3D) & 4 & 2 weeks & Retro-AAV-tdTomato $\left(3.8 \times 10^{12} \mathrm{GC} / \mathrm{ml}\right)$ & $3 D$ \\
\hline Dual label (cervical/lumbar) (3D) & 4 & 4 weeks & $\begin{array}{l}\text { Retro-AAV-tdTomato }\left(3.8 \times 10^{12} \mathrm{GC} / \mathrm{ml}\right) \\
\text { Retro-AAV-EGFP }\left(3.9 \times 10^{12} \mathrm{GC} / \mathrm{ml}\right)\end{array}$ & $3 \mathrm{D}$ \\
\hline DREADD behavior, wild-type & 12 & Behavior weeks $2-4$; killed at 4 weeks & $\begin{array}{l}\text { Retro-AAV-CRE }\left(8.1 \times 10^{12} \mathrm{GC} / \mathrm{ml}\right) \\
\text { Retro-AAV-Flex-Gi-DREADD }\left(9.0 \times 10^{12} \mathrm{GC} / \mathrm{ml}\right)\end{array}$ & $2 D$ \\
\hline DREADD behavior, CaMKIla-Cre & 6 & Behavior weeks $2-4$; killed at 4 weeks & Retro-AAV-Flex-Gi-DREADD $\left(9.0 \times 10^{12} \mathrm{GC} / \mathrm{ml}\right)$ & $2 D$ \\
\hline
\end{tabular}

Statistical comparisons of transduction efficiencies between all suprapsinal populations are provided in Table 1-2, available at https://doi.org/10.1523/JNEUROSCI.1196-18.2018.t1-2.

A

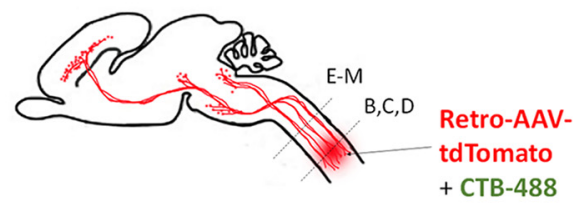

3 days
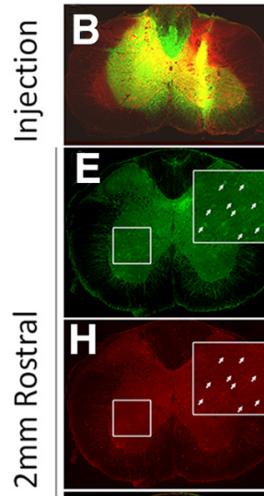

7 days
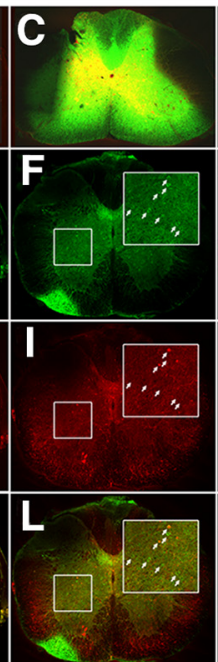

14 days
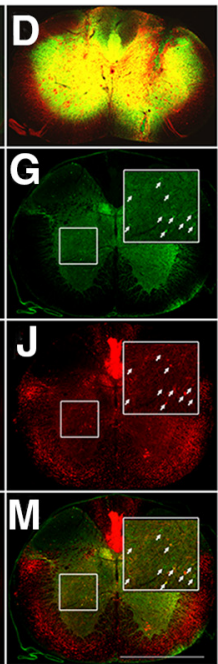

$\mathrm{N}$
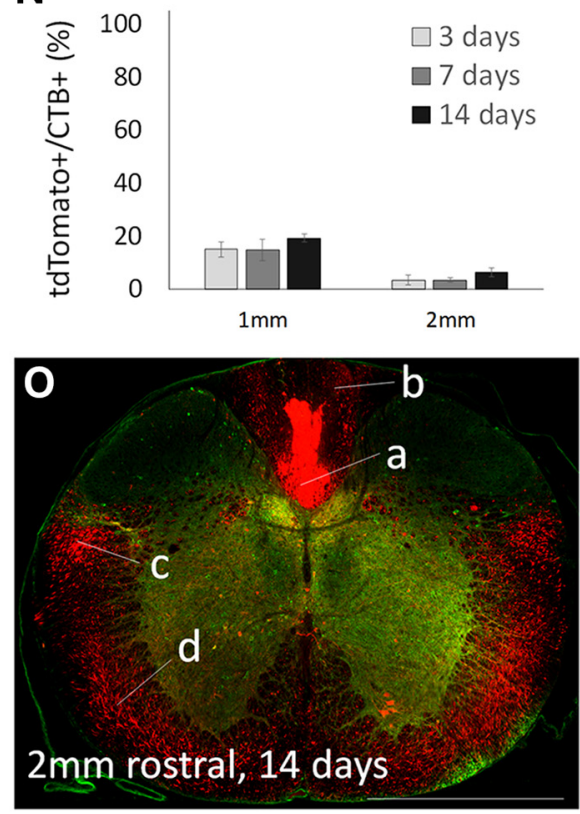

a) Corticospinal tract b) ascending sensory

c) rubrospinal d) lateral spinocerebellar

Figure 1. AAV2-Retro shows limited retrograde transduction of cervical propriospinal neurons. $A$, Mixed AAV2-Retro-tdTomato and CTB-488 were injected bilaterally to C $4 / 5$ gray matter, and transverse spinal sections at the injury site and 1 or $2 \mathrm{~mm}$ rostral were examined 3,7 , or $14 \mathrm{~d}$ later. $\boldsymbol{B}-\boldsymbol{D}$, Transverse sections at the level of spinal injection show readily detectable CTB-488 and tdTomato. $\boldsymbol{E}-\mathbf{G}$, In cervical spinal cord $2 \mathrm{~mm}$ rostral to the injection, (TB-488 retrogradely labels cell bodies that project axons to the injection site (arrows). $\boldsymbol{H}-\boldsymbol{M}$, tdTomato signal is rarely detectable in CTB-488 + cell bodies, indicating low transduction. $N$, Quantification of tdTomato detection in CTB-488 + cell bodies showed that $<20 \%$ of propriospinal neurons projecting to C $4 / 5$ were transduced $1 \mathrm{~mm}$ rostral to the injury, and $<10 \%$ at $2 \mathrm{~mm} . \mathbf{0}$, By $14 \mathrm{~d}$ postinjection, tdTomato signal is apparent in locations corresponding to ascending and descending axon tracts. $N>100$ individual cells from each of at least four animals at each time point. Error bars show SEM. Scale bars, $1 \mathrm{~mm}$. Statistical comparisons of transduction efficiencies between supraspinal populations are provided in Figure 1-1, available at https://doi.org/10.1523/JNEUROSCI.1196-18.2018.f1-1.

bearing locomotion but entirely non-plantar placement, 2 = no weightbearing steps, but forelimbs make sweeping motions for forward propulsion, 1 = no weight-bearing steps, forelimbs make small movements that do not move body, $0=$ forelimbs pinned with no discernible movement. In addition, transgenic CaMKIIa-Cre mice [The Jackson Laboratory, B6.Cg-Tg(CaMKIIa-cre)T29-1Stl/J] were tested on modified horizontal ladder task in which animals walked atop a wheel with variably spaced rungs (average $1.0 \mathrm{~cm}$ gap, range $0.5-1.5 \mathrm{~cm}$ ). Animals were confined to the apex of the wheel, which rotated beneath them at a fixed speed of $0.78 \mathrm{~m} / \mathrm{min}$. After one full rotation, data were collected for the next three rotations. Each right forelimb step was scored as correct (step centered on rung, which is grasped), wrist or toe placement errors (forelimb takes weight but is placed too far forward or back, such that rung is not grasped), or complete miss (wrist breaks the plane of the rungs). Animals were pretrained on the task, and then tested in the absence of clozapine administration or $15 \mathrm{~min}$ after intraperitoneal clozapine injection $(0.1 \mathrm{mg} / \mathrm{kg})$. All behavioral scoring was performed by individuals blind to experimental treatment.
Tissue clearing and imaging. Animals were transcardially perfused with $0.9 \%$ saline and $4 \%$ paraformaldehyde solutions in $1 \times$-PBS (15710, Electron Microscopy Sciences). Whole brains and spinal cords were dissected and fixed overnight in $4 \%$ paraformaldehyde at $4{ }^{\circ} \mathrm{C}$ and washed three times in PBS, pH 7.4, and stored in PBS. The dura was carefully and completely removed as residual dura can trap bubbles that prevent effective light-sheet microscopy. Samples were incubated on a shaker at room temperature in 50, 80, and $100 \%$ peroxide-free tetrahydrofuran (THF; Sigma-Aldrich, 401757) for $12 \mathrm{~h}$ each. Peroxides were removed from THF by passing $100 \%$ THF through a chromatography column filled with basic activated aluminum oxide (Sigma-Aldrich, 199443) as previously described (Ertürk et al., 2012; Soderblom et al., 2015). The next day, samples were transferred to BABB solution (1:2 ratio of benzyl alcohol, Sigma-Aldrich, 305197; and benzyl benzoate, Sigma-Aldrich, B6630) for at least $3 \mathrm{~h}$. After clearing, samples were imaged within $48 \mathrm{~h}$ by light-sheet microscopy (Ultramicroscope, LaVision BioTec). The ultramicroscope uses a fluorescence macro zoom microscope (Olympus MVX10) with a $2 \times$ Plan Apochromatic zoom objective (NA 0.50). Image 
A

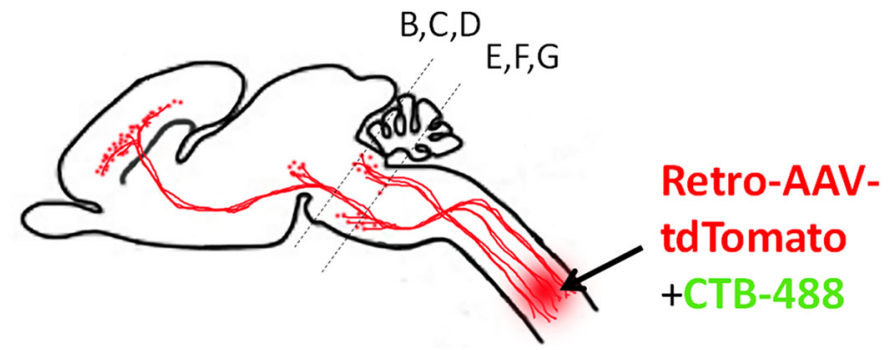

\section{3 days}
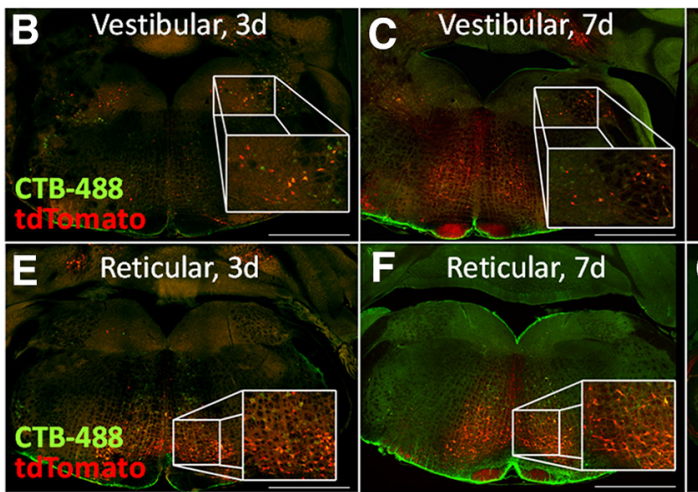

H
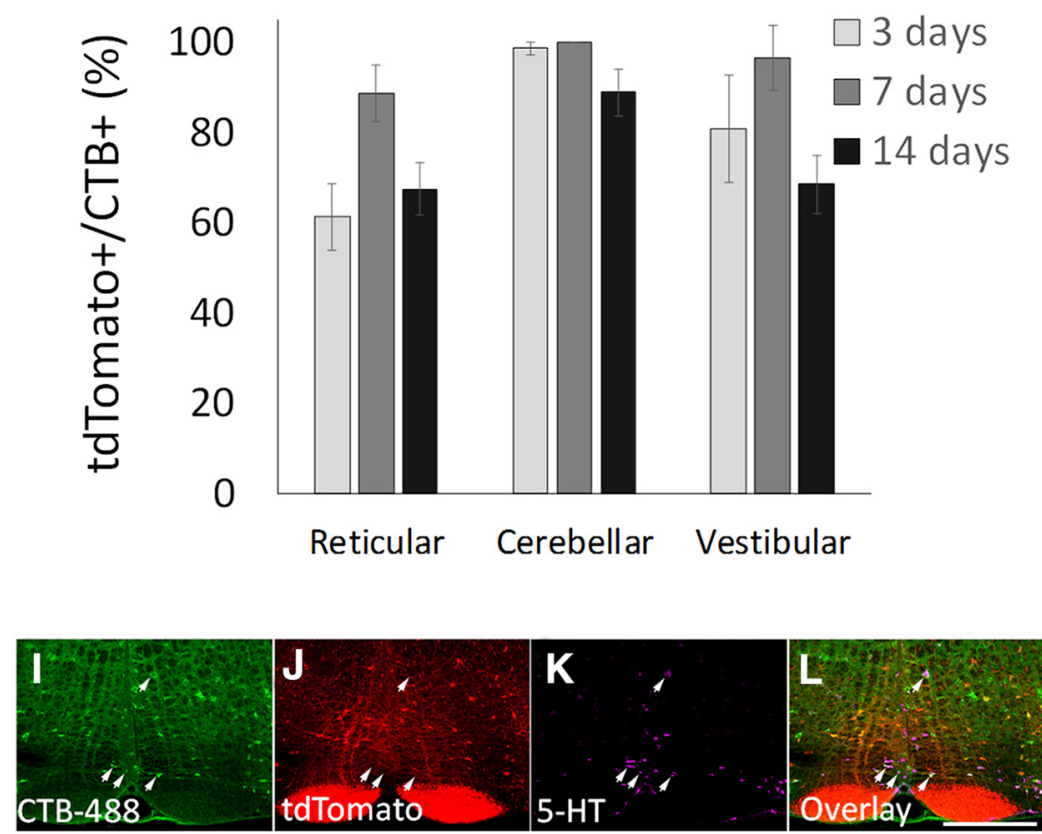

Figure 2. AAV2-Retro effectively transduces brainstem-spinal projection neurons. $A$, Mixed AAV2-Retro-tdTomato and CTB488 were injected bilaterally to $(4 / 5$ gray matter, and transverse sections of brainstem examined 3,7 , or $14 \mathrm{~d}$ later. In vestibulospinal $(\boldsymbol{B}-\boldsymbol{D})$, reticulospinal $(\boldsymbol{E}-\boldsymbol{G})$, and cerebellar-spinal $(\boldsymbol{E}-\boldsymbol{G})$ populations, spinally projecting neurons identified by $(\mathrm{TB}-488$ also express tdTomato as early as $3 \mathrm{~d}$ postinjection. $\boldsymbol{H}$, Quantification of the percentage of CTB-488 + neurons that are virally transduced shows a majority of vestibulospinal, reticulospinal, and cerebellar-spinal neurons are retrogradely labeled. $N>100$ individual cells from each of at least four animals at each time point. No significant differences between groups existed $(p>0.05$, two-way ANOVA with post hoc Tukey's). I-L, Two weeks after spinal injection of mixed (TB-488 and AAV2-Retro-tdTomato, transverse brainstem sections were immunostained for 5HT. Raphe-spinal neurons (arrows) were identified by CTB-488 $(I)$ to confirm an axon projection to the $C 4 / 5$ injection site, and expression of $5 \mathrm{HT}(\boldsymbol{K})$. These neurons were invariably negative for tdTomato, although nearby non-serotonergic neurons were effectively transduced. Scale bars: $B-G, 1 \mathrm{~mm} ; \boldsymbol{I}-\boldsymbol{L}, 500 \mu \mathrm{m}$. Error bars show SEM. analysis and 3D reconstructions were performed using Imaris v8.4.2 software (Bitplane) after removing autofluorescence using the Imaris Background Subtraction function with the default filter width so that only broad intensity variations were eliminated. Artifact and nonspecific fluorescence surrounding the brain was segmented and removed using the automatic isosurface creation wizard based upon absolute intensity. Some of the cleared brains were also sliced to generate thick sections of 500-1000 micrometers and imaged on a FV1000 (Olympus) laser scanning microscope. Movies were generated using Imaris and merged in Photoshop CS6.

Experimental design and statistical analysis. An initial in vivo time course experiment was initiated with 15 adult female mice, killed in equal groups at 3,7 , and $14 \mathrm{~d}$ postinjection. One animal in the $3 \mathrm{~d}$ group was lost to mortality, and one animal in the $7 \mathrm{~d}$ group was excluded on the basis of a mistargeted injection (no CTB-488 label detected in the spinal cord). The in vivo spinal injury experiment was initiated with five adult female mice in each group (injured vs uninjured). One injured animal was lost to mortality and one uninjured animal was excluded on the basis of mistargeted injections, leaving four animals in each group. To compare efficiency of transduction across all populations, the percentage of dual CTB/AAV labeling was quantified from at least 100 cells in each animal, and then compared across groups by two-way ANOVA with post hoc Tukey's. To compare injured and uninjured animals, ANOVA with post hoc Sidak's tested for differences in transduction efficiency. For tissue clearing, four animals received cervical injection of AAV2-Retro-tdTomato to cervical spinal cord and were killed $14 \mathrm{~d}$ later, and four adult female mice received both cervical AAV2-Retro-EGFP and lumbar AAV2-RetrotdTomato and were killed 4 weeks later. DREADD experiments were initiated with five wild-type and five CaMKIIa-Cre female mice injected with AAV2-Retro-DREADD constructs, and three uninjected wild-type females. One CaMKIIa-Cre was lost to mortality. Slip rates in the presence or absence of clozapine stimulation on the ladder task were compared by two-tailed paired $t$ tests. An overview of animal numbers and experimental variables is provided in Table 1, and a summary of statistical results is provided in Table 1-2, available at https://doi.org/10.1523/JNEUROSCI. 1196-18.2018.t1-2.

\section{Results}

AAV2-Retro effectively transduces reticulospinal, rubrospinal, and corticospinal neurons

AAV serotypes vary widely in their tropism for different types of neurons and their onset of transgene expression (Castle et al., 2016). This appears to be particularly true for retrograde transduction from the spinal cord, because prior tests showed higher efficiency in propriospinal 
and brainstem populations compared with CST neurons (Jara et al., 2012; Klaw et al., 2013). To comprehensively profile the efficiency and time course of retrograde transduction by AAV2Retro in supraspinal populations, 15 adult female mice were injected with a mixture of the retrograde tracer CTB488 and AAV2-Retro-tdTomato, bilaterally to cervical spinal cord. Animals were killed at 3, 7, or $14 \mathrm{~d}$ postinjection and complete series of $120 \mu \mathrm{m}$ sections of the brain and cervical spinal cord was collected. CTB-488 is a widely used retrograde tracer that efficiently labels supraspinal neurons and was used as a positive control to mark neurons that projected axons to the site of injection. Sections were imaged by confocal microscopy and transduction efficiency was quantified by the percentage of CTB-488+ neurons in each population that coexpressed tdTomato. To avoid sampling bias, sections for imaging were preselected on the basis of their position in the rostral-caudal series. All CTB-488-positive cells were identified and marked in each section, after which the corresponding tdTomato image was examined to quantify the percentage of CTB-488+ cells that coexpressed tdTomato. A summary and statistical comparison of transduction efficiency across all populations and time points is provided in Table 1-2, available at https://doi.org/10. 1523/JNEUROSCI.1196-18.2018.t1-2 and Figure 1-1, available at https://doi. org/10.1523/JNEUROSCI.1196-18.2018. f1-1 and detailed descriptions of selected nuclei are provided in the next section.

Injection site and cervical propriospinal neurons

Spinal cord sections were collected from the site of injection and at 1 and $2 \mathrm{~mm}$ rostral. At the injection site CTB488 and tdTomato signals were intense at all time points, confirming appropriate targeting and transduction of cell bodies directly exposed to AAV2Retro (Fig. $1 B-D$ ). At locations 1 and 2 $\mathrm{mm}$ rostral to the site of injection, numerous cell bodies were labeled with CTB-488, indicating retrograde transport of CTB-488 (Fig. 1E-G). Of these, $<20 \%$ at $1 \mathrm{~mm}$ and $<10 \%$ at $2 \mathrm{~mm}$ coexpressed tdTomato at any time point (Fig $1 H-N ; 1 \mathrm{~mm}$ : 10, 14, and $19 \%$ at 3,7, and $14 \mathrm{~d} ; 2 \mathrm{~mm} \mathrm{3,3}$, and $6 \%$ at 3, 7, and $14 \mathrm{~d}$ ). Interestingly, despite the low transduction in spinal neurons, by $14 \mathrm{~d}$ tdTomato label was detected in white matter locations corresponding to descending tracts, including areas that contain

A

$\mathbf{T}$
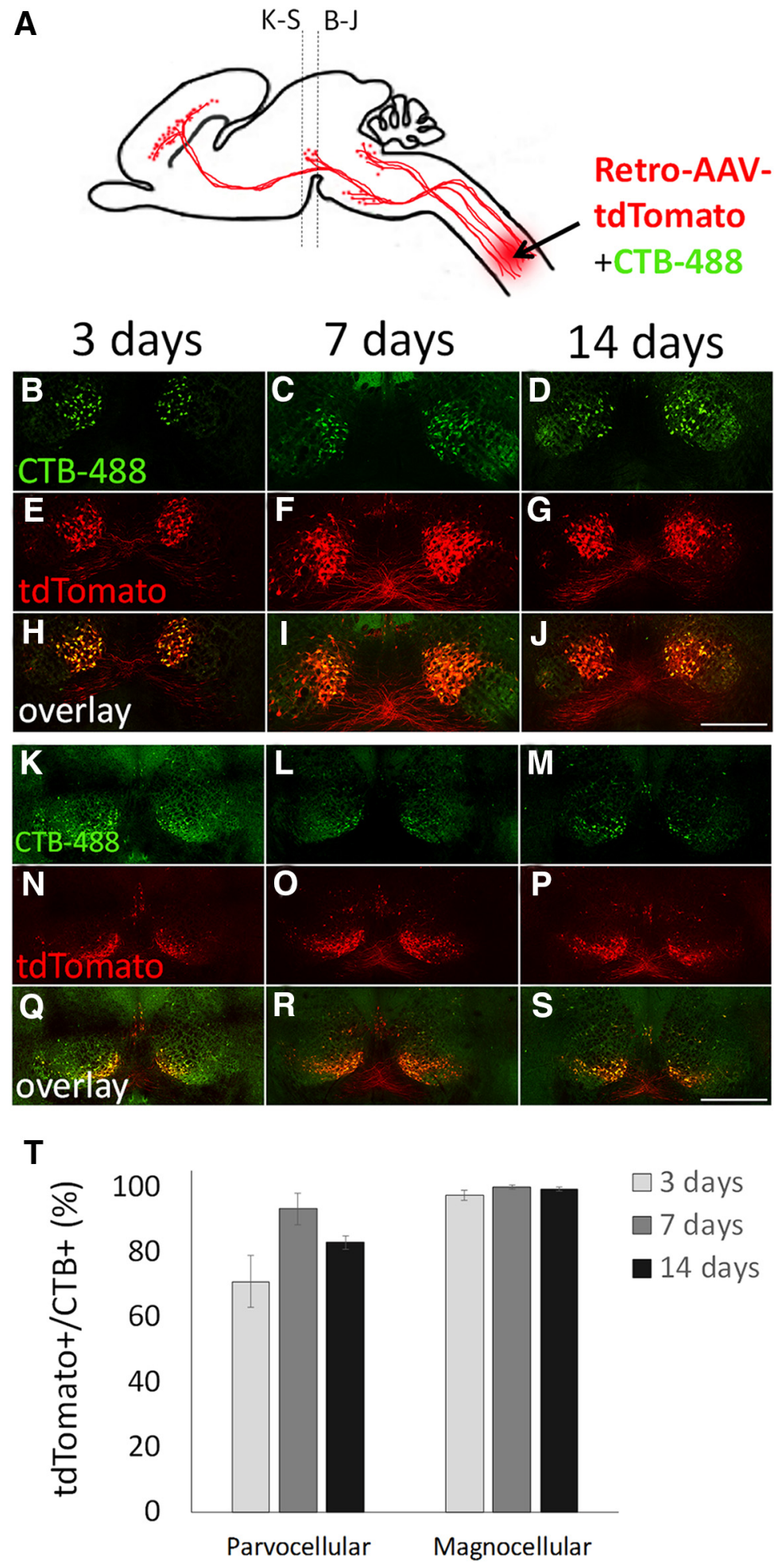

Figure 3. Effective retrograde transduction of rubrospinal neurons. $A$, Mixed (TB-488 and AAV2-Retro-tdTomato were injected to cervical spinal cord and transverse sections of midbrain prepared 3,7 , or $14 \mathrm{~d}$ later. $\boldsymbol{B}-\boldsymbol{J}$, The magnocellular population and $(\boldsymbol{K}-\boldsymbol{S})$ the parvocellular. $T$, A majority of (TB-488 + neurons in both parvo- and magnocellular nuclei were colabeled with tdTomato, indicating rapid and effective transduction. Groups did not differ significantly ( $p>0.05$, two-way ANOVA with post hoc Tukey's). $N>100$ individual cells from each of at least four animals at each time point. Scale bars, $500 \mu \mathrm{m}$. Error bars show SEM.

corticospinal and rubrospinal axons (Fig. 1O). This axonal labeling hinted at more effective transduction in higher supraspinal populations, which we confirm below. These data show successful targeting of injections and transduction at sites of spinal injection, but indicate relatively low retrograde 
A

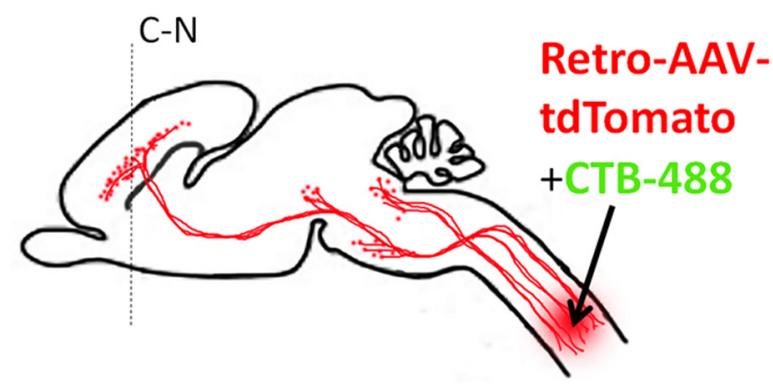

3 days

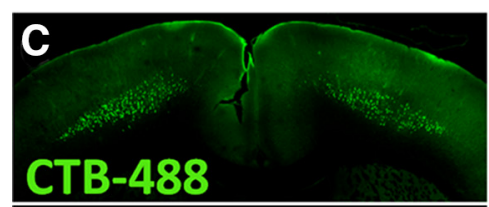

$\mathbf{F}$

tdTomato
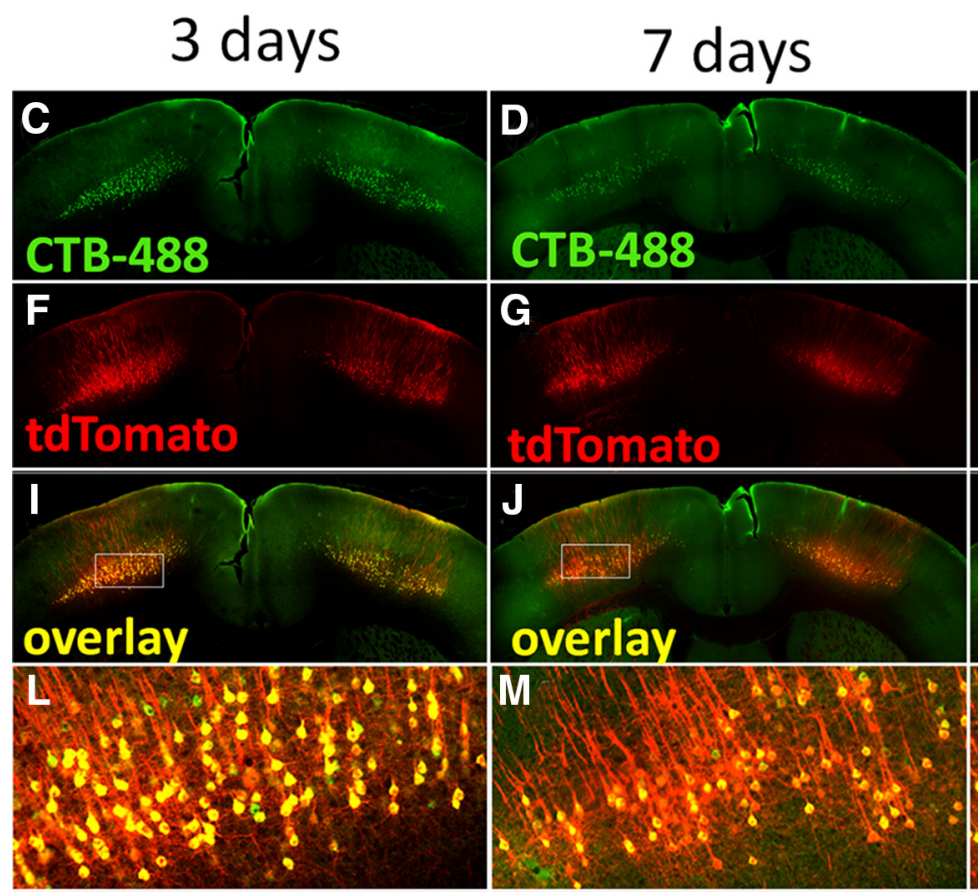

B

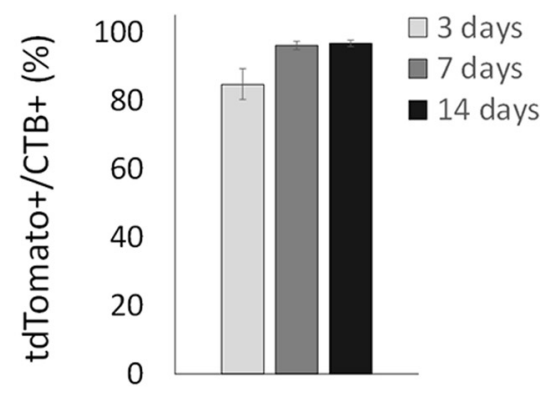

14 davs
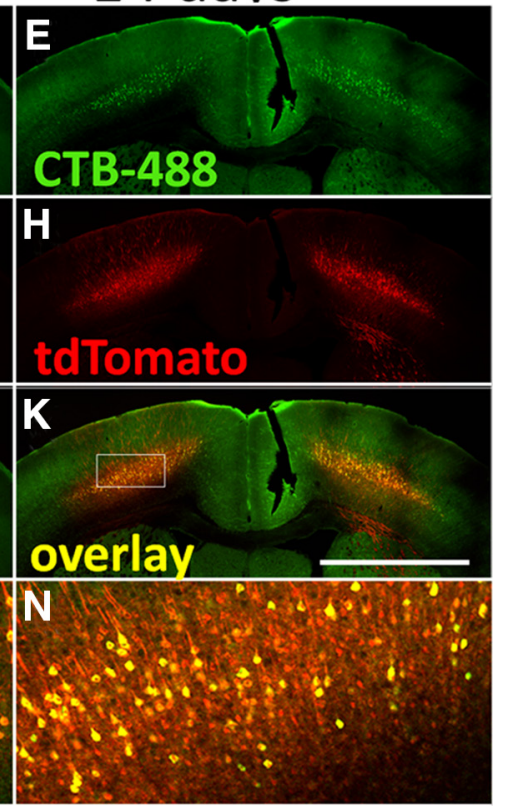

Figure 4. Effective retrograde transduction of corticospinal tract neurons. $\boldsymbol{A}, \boldsymbol{B}$, Adult mice received cervical injection of mixed AAV2-Retro-tdTomato and CTB-488, and the percentage of double-labeled cells quantified in transverse sections of cortex 3, 7, or 14 d later. $\mathbf{C}-\boldsymbol{E}$, (TB-488 identifies (ST neurons. $\boldsymbol{F}-\boldsymbol{H}$, tdTomato is readily detectable as early as $3 \mathrm{~d}$ postinjection). $\boldsymbol{I}-\boldsymbol{N}$, 0 verlays show the great majority of (TB-488+ cells also express tdTomato, indicating efficient retrograde transduction. Groups did not differ significantly ( $p>0.05$, ANOVA with post hoc Sidak's). N $\geq 4$ animals per time point, $>100$ cells/section, 3 sections per animal. Scale bar, 2 mm. Error bars show SEM.

transduction efficiency in nearby propriospinal populations in upper cervical spinal cord.

\section{Brainstem and cerebellar neurons}

Spinal projection neurons throughout the brainstem were labeled by CTB-488. Based on anterior/posterior position in the sequence of sections and nearby neuroanatomical landmarks, we identified clusters of CTB-488-positive cells corresponding to reticulospinal, cerebellospinal, and vestibulospinal nuclei (Fig. 2A-G). By $3 \mathrm{~d}$ postinjection, AAV2-Retro-tdTomato signal was readily detectable in a majority of these cells [reticulospinal: $61.2( \pm 7.4$ SEM)\%, 88.6 ( \pm 1.6 SEM $) \%$, and 67.4 ( \pm 5.8 SEM $) \%$, cerebellospinal: $98.6( \pm 1.4$ SEM)\%, $100( \pm 0$ SEM $) \%$, and $88.8( \pm 5.2$ SEM)\%, vestibulospinal: 80.8 ( \pm 1.4 SEM $) \%, 96.5 \quad( \pm 0.9$ $\mathrm{SEM}) \%$, and 68.5 ( $\pm 6.4 \mathrm{SEM}) \%$, at 3, 7, and $14 \mathrm{~d}$ postinjection, respectively; Fig. $2 \mathrm{H}$ ]. Next, using $5 \mathrm{HT}$ immunohistochemistry, we identified raphe neurons located near the medullary ventral midline (Fig. $2 I-L$ ). In striking contrast to other brainstem-spinal populations, tdTomato signal was absent from 5-HT-positive raphe neurons at all time points, including cells marked by CTB-488 signal that confirmed axonal projection to the site of spinal injection (Fig. $2 L$, arrows). These data indicate cell-type tropism that prevents ef- fective transduction of serotonergic neurons. Consistent with this, the initial characterization of AAV2-Retro indicated preferential retrograde transduction of glutamatergic neurons and limited transduction of dopaminergic neurons (Tervo et al., 2016). In summary, these results indicate that AAV2-Retro effectively and rapidly transduces most brainstem-spinal populations, with the notable exception of raphespinal projection.

\section{Red nucleus}

The red nucleus spans the boundary of the diencephalon and midbrain and is composed of two distinct populations of neurons, the rostral parvocellular, and the caudal magnocellular. Both populations project axons to the spinal cord and, as expected, were effectively labeled by retrograde CTB-488 (Fig. $3 A-D, K-M$; Liang et al., 2012). In the magnocellular nucleus virtually all spinally projecting neurons were transduced, with $97.5( \pm 1.5 \mathrm{SEM}) \%, 100( \pm 0.7$ SEM)\%, and 99.3 ( $\pm 0.5 \mathrm{SEM}) \%$ colabeled at 3,7 , and $14 \mathrm{~d}$ postinjection (Fig. $3 E-J, T$ ). In the parvocellular division, $70.9( \pm 8.0$ SEM $) \%, 93.3( \pm 4.9$ SEM $) \%$, and 82.9 ( \pm 2.0 SEM $) \%$ of CTB- $488+$ cells expressed tdTomato at 3,7 , and 14 d postinjection, respectively (Fig. $3 N-T$ ). Thus spinally injected AAV2-Retro effectively transduces cell bodies in the red nucleus. 


\section{Corticospinal}

CST neurons reside in layer $\mathrm{V}$ of sensorimotor cortex and provide the only direct input from cortex to spinal cord. Until recently, retrograde vectors have displayed a limited ability to transduce CST neurons. We examined transverse cortical sections between $3 \mathrm{~mm}$ anterior and $2 \mathrm{~mm}$ posterior to bregma, which spans all regions of cortex that project axons to the spinal cord (for a more complete description of distinct CST populations, see discussion of Movie 1 in the section Tissue Clearing and 3D Reconstruction). As expected, retrograde CTB-488 was readily detected in CST cell bodies at all time points (Fig. $4 A-E$ ). Virally expressed tdTomato was expressed in $>80 \%$ of CTB- $488+$ cell bodies by just $3 \mathrm{~d}$ postinjection, and in $>95 \%$ of CTB- $488+$ cell bodies by 7 and $14 \mathrm{~d}$ (Fig. $4 F-N)$. As noted earlier, tdTomato signal emerged in CST projection tracts in the brainstem by $7 \mathrm{~d}$ postinjection (Fig. 2C,F), and in the spinal CST by $14 \mathrm{~d}$ (Fig. 1O). These data indicate that cervical injection of AAV2-Retro results in expression in most CST neurons within $3 \mathrm{~d}$, and that within 2 weeks fluorophore expression and transport are sufficient to detect the projecting axons in cervical spinal cord.

\section{Retrograde transduction remains effective following cervical spinal injury}

A prerequisite for potential therapeutic utility is that retrograde vectors display effective retrograde transduction when applied to axons after injury. We therefore tested AAV2-Retro administration after cervical spinal injury. Four adult female mice received unilateral dorsal hemisections of C4/5 spinal cord, which completely severs the dorsal and dorsal-lateral CST axons on the right side of the spinal cord. Immediately after injury, animals received bilateral injections of mixed AAV2-Retro-tdTomato and CTB488 to $\mathrm{C} 4$ spinal cord, $0.5 \mathrm{~mm}$ rostral to the injury. As control, four additional animals received AAV2-Retro-tdTomato/CTB-488 injections without injury. Animals were killed at $28 \mathrm{~d}$ postinjection and a complete series of $120 \mu \mathrm{m}$ sections of the brain was collected, along with sagittal sections of the spinal injury site. Immunohistochemistry for GFAP at the site of spinal injury confirmed lesion depth in all animals, and in the dorsal cord tdTomato and CTB-488 signal was confined to tissue rostral to the injury, indicating complete ablation of dorsal axon tracts (Fig. $5 A-E)$. As expected from this partial injury, tdTomato + axons continued uninterrupted in ventral tracts, indicating likely sparing of other supraspinal neurons that project axons ventrally. We therefore focused our analysis on the CST neurons, a population unequivocally affected by dorsal injury. As expected, in uninjured animals CTB-488 retrogradely labeled cortical cell bodies, and the great majority of these expressed tdTomato (right cortex: 93.96 ( \pm 2.9 SEM)\%, left cortex 93.9 ( \pm 2.0 SEM)\%; Fig. 5F, $H$ ). Similarly, in the right (spared) cortex of animals that received unilateral spinal transection, transduction efficiency was 90.3 $( \pm 5.7 \%$; Fig. $5 F, H)$. In the left (injured) cortex, efficiency was $98.8( \pm 1.1 \%)$, not significantly different from either contralateral cortex or uninjured animals $(p=0.043$, two-way ANOVA with post hoc Tukey's; Fig. 5G,H; Table 1-2, available at https:// doi.org/10.1523/JNEUROSCI.1196-18.2018.t1-2). Consistently, the absolute numbers of tdTomato + cells counted in five tissue sections was similar in right and left cortex (right: $339.3( \pm 71.7$ SEM), left: 400.7 ( $\pm 42.0 \mathrm{SEM} ; N=3, p=0.51$, paired $t$ test). Thus AAV2-Retro-tdTomato is effective in transducing CST neurons when applied acutely after a spinal injury.
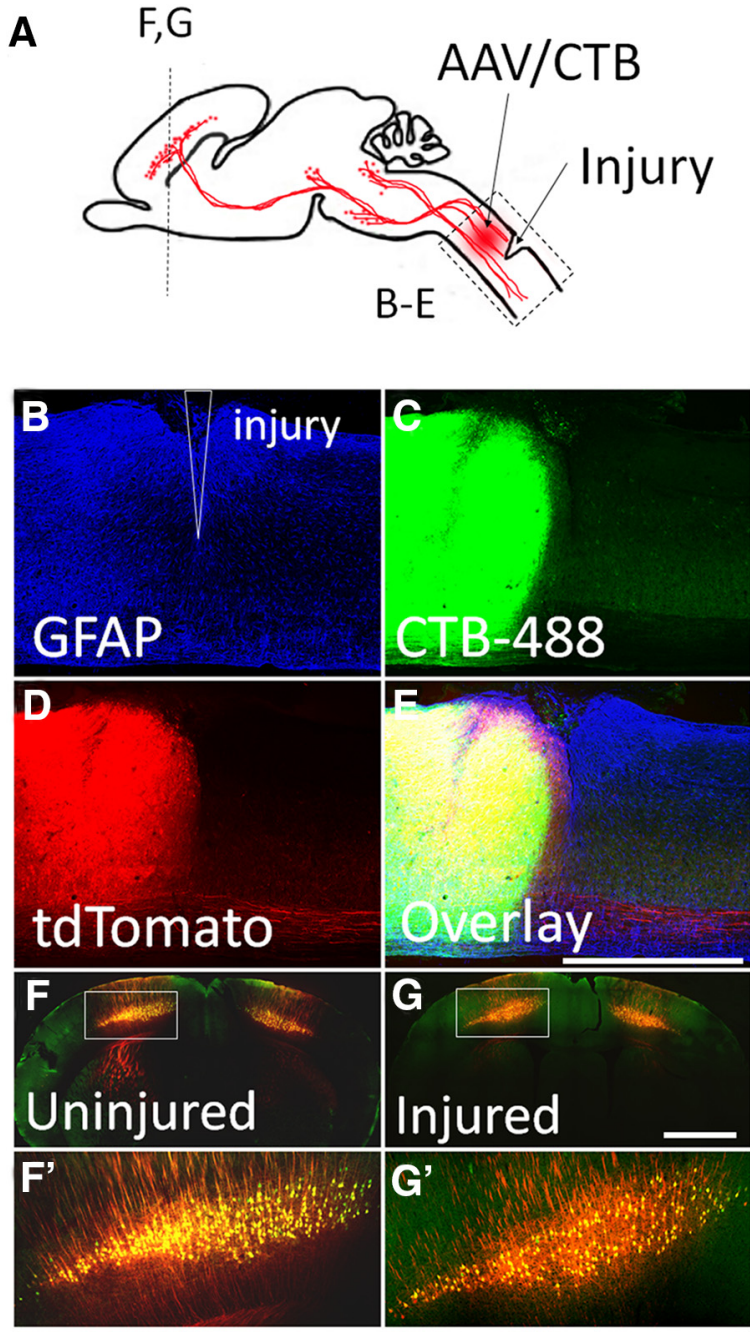

H

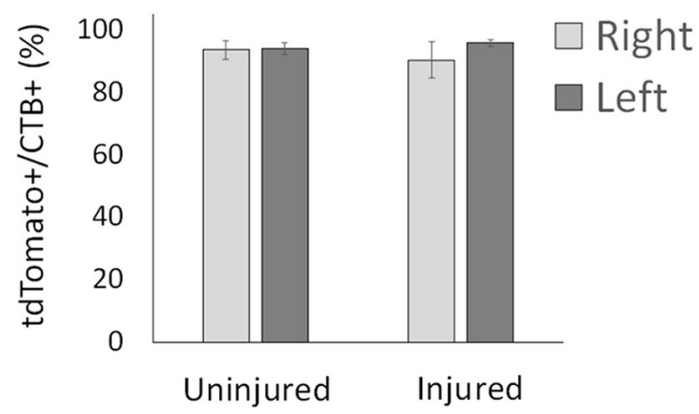

Figure 5. Effective retrograde transduction of corticospinal tract neurons after spina injury. $A$, Adult female mice received unilateral cervical dorsal hemisection or sham injury, followed by bilateral injection of mixed Retro-AAV-tdTomato and CTB-488. $\boldsymbol{B}-\boldsymbol{E}$, In sagittal sections of spinal cord $28 \mathrm{~d}$ postinjection, GFAP immunohistochemistry $(\boldsymbol{B})$ and the absence of (TB-488 ( $($ ) and tdTomato $(\boldsymbol{D})$ signal caudal to the injury confirmed complete dorsal transections. $\boldsymbol{F}, \mathbf{G}$, Coexpression of CTB-488 and tdTomato was high in CST neurons in injured animals $(\boldsymbol{F})$ and in both cortices of animals that received unilateral spinal injury (G). $\boldsymbol{H}$, Quantification of coexpression rates showed no difference in the left cortex of injured animals, the location of axotomized CST cell bodies ( $p=0.82$, two-way ANOVA with post hoc Tukey's). $N=4$ animals per group, $>200$ individual cells quantified per animal. Error bars show SEM. Scale bars, $1 \mathrm{~mm}$

Tissue clearing and $3 \mathrm{D}$ reconstruction reveals spatial organization of descending spinal inputs

Advances in tissue clearing and 3D microscopy now enable the visualization of neurons and their projections in intact tissue. We 

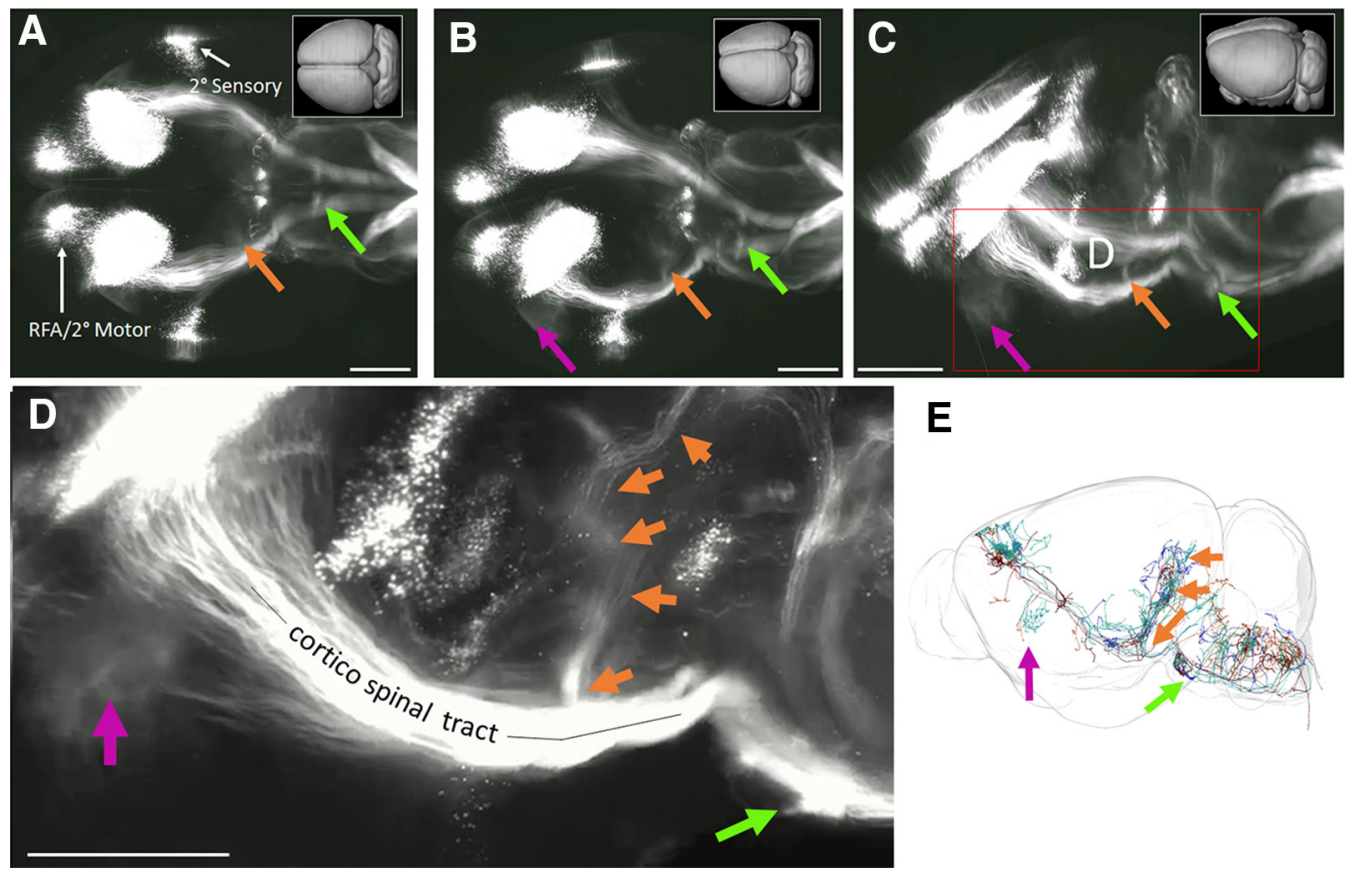

E

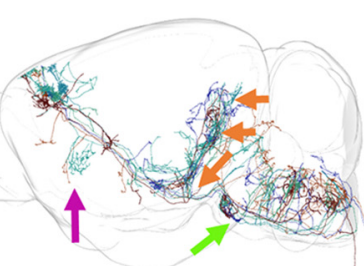

Figure 6. AAV2-Retro and 3D imaging reveal CST cell body distribution, axon trajectories, and collateralization. $\boldsymbol{A}-\boldsymbol{D}$, Adult mice received bilateral cervical injection of AAV2-Retro-tdTomato. Two weeks later brains were optically cleared by 3 DISCO and imaged with light-sheet microscopy. Images showed rotated views of whole brain, with rostral to the left and initially viewed from the dorsal surface in $\boldsymbol{A}$. Three distinct groups of CST cell bodies are apparent, and to clear points of collateral branching from the main CST are visible (arrows). $\boldsymbol{E}$, Individual tracings of CST neurons from the Mouselight Neuron Browser. Consistent with the 3D reconstruction, collateral branches to tectal areas (orange arrow) and to Basilar Pontine Nuclei (green arrow) are visible. Videos of cleared brains are available in Movies 1,2,3, and 4. Scale bars, $1 \mathrm{~mm}$.

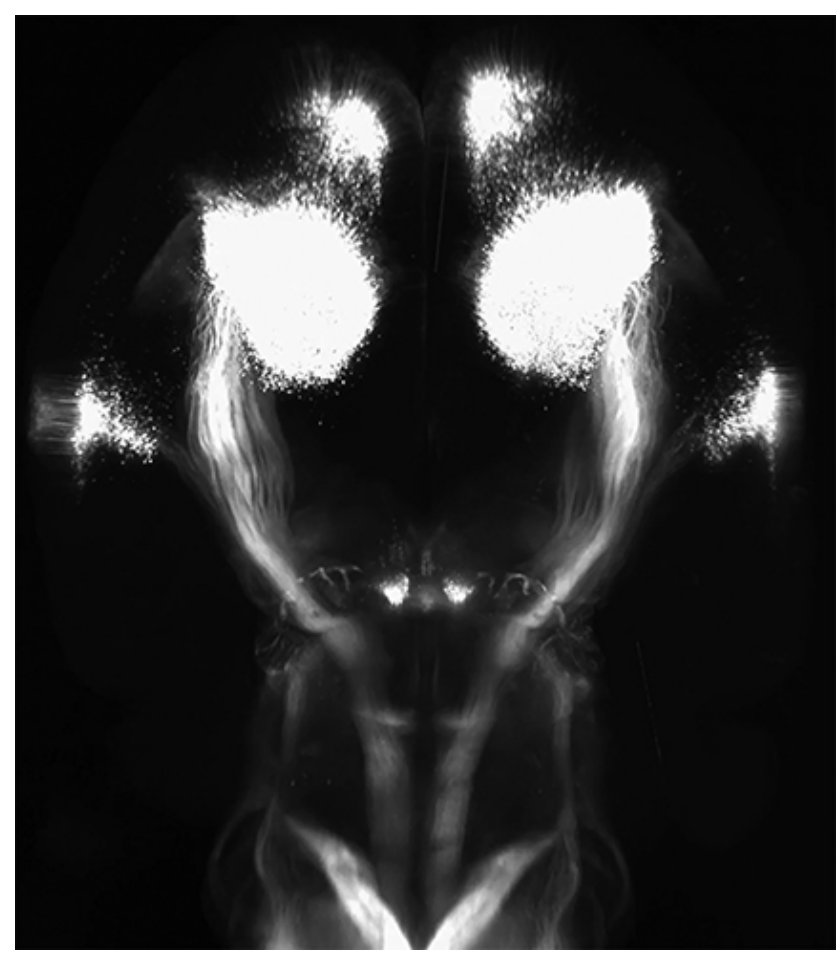

Movie 1. 3D visualization of corticospinal projections and collateral branches. Adult mice received bilateral cervical injection of AAV2-RetrotdTomato. Two weeks later brains were optically cleared by $3 \mathrm{DISCO}$ and imaged with light-sheet microscopy. This video illustrates the overall distribution of CST projection neurons and points of collateralization of the descending CST.

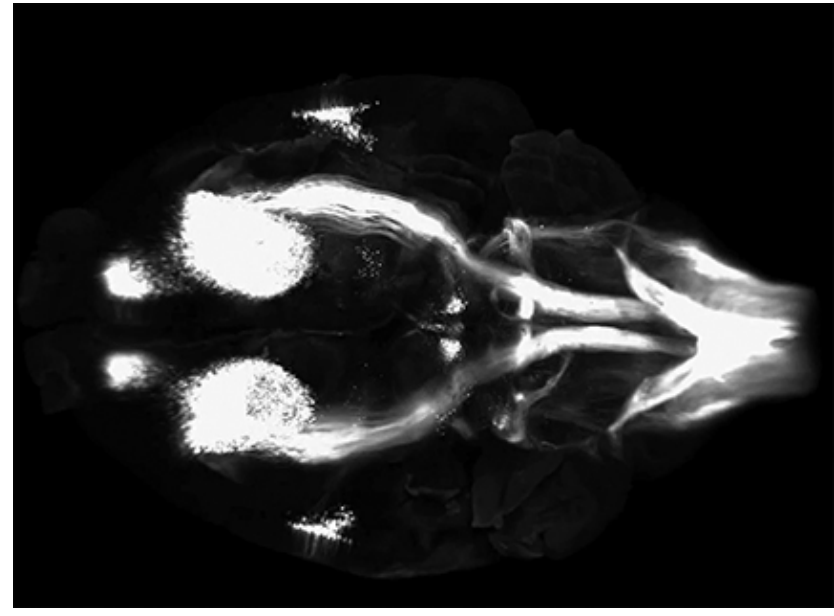

Movie 2. Alternative 3D visualization of corticospinal projections and collateral branches. Adult mice received bilateral cervical injection of AAV2-Retro-tdTomato. Four weeks later brains were optically cleared by $3 \mathrm{DISC} 0$ and imaged with light-sheet microscopy. This video provides additional details regarding the collateralization of descending CST axons.

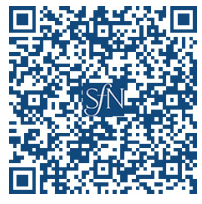

therefore injected AAV2-Retro-tdTomato bilaterally to cervical spinal cord, and then 2 weeks later performed tissue clearing with and 3D-imaging of brain tissue (Soderblom et al., 2015; Bray et al., 2017). We imaged tissue using only the native tdTomato fluorescent emission of light, without antibody amplification. The original 3DISCO based clearing method can cause progressive loss of fluorescent signal that becomes substantial after $\sim 48 \mathrm{~h}$, and therefore all imaging was performed within $24 \mathrm{~h}$ of clearing (Soderblom et al., 2015). Consistent with the section-based his- 


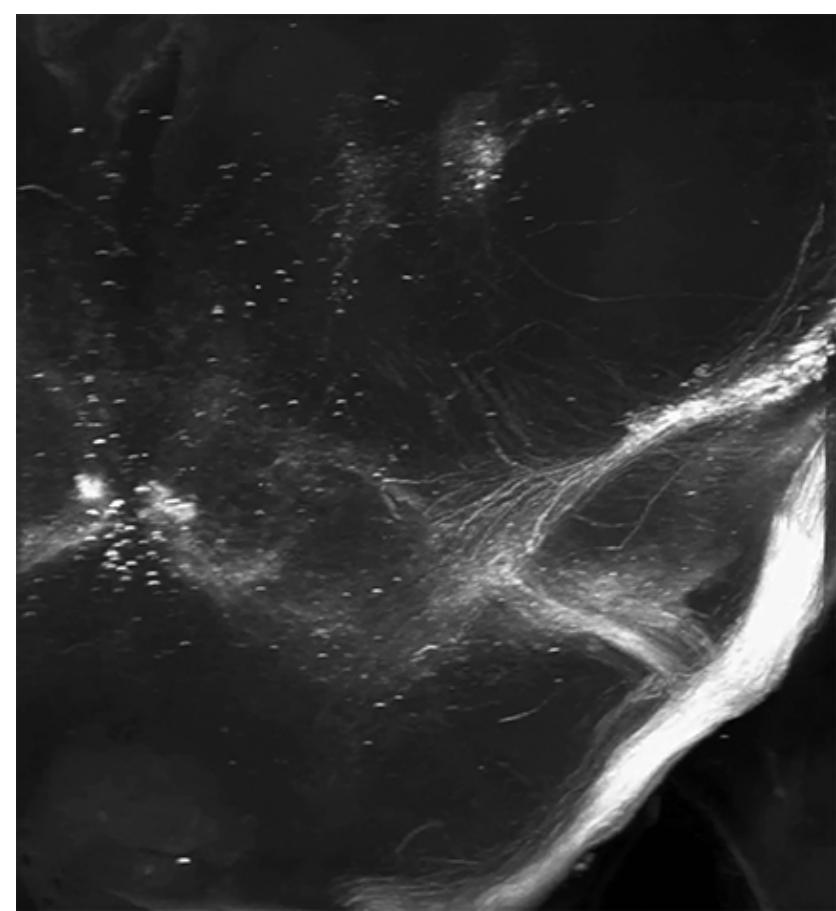

Movie 3. 3D visualization of CST-tectal collaterals. Adult mice received bilateral cervical injection of AAV2-Retro-tdTomato. Four weeks later brains were optically cleared by 3DISCO and imaged with lightsheet microscopy. This video provides a high-resolution visualization of collaterals emanating from CST axons and extending toward pre-tectal targets.

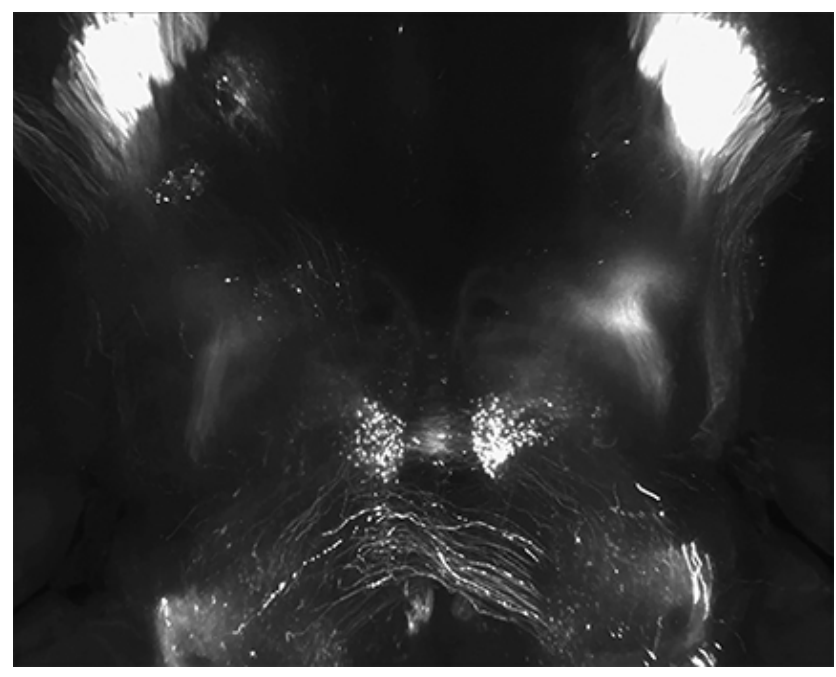

Movie 4. 3D visualization of corticospinal-red nucleus collaterals. Adult mice received bilateral cervical injection of AAV2-RetrotdTomato. Four weeks later brains were optically cleared by $3 \mathrm{DISCO}$ and imaged with light-sheet microscopy. This video illustrates the potential extension of CST collaterals to the red nucleus.

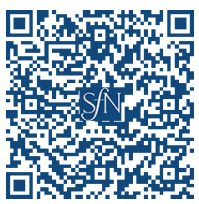

tology discussed above, 3D imaging of intact tissue revealed widespread fluorophore expression in supraspinal nuclei in the brain and in descending axon tracts. In addition, the 3D imaging provided new insights by revealing anatomical features of supraspinal input that were difficult to detect in the $2 \mathrm{D}$ tissue sections.
First, 3D imaging clarified the distribution of corticospinal tract neurons into three distinct groups: a large central mass corresponding to primary sensorimotor cortex, a distinct group located rostrally, corresponding to secondary motor cortex or rostral forelimb area, and a caudal/lateral group corresponding to secondary sensory cortex (Fig. 6A-C; Movie 1. Axons from each area could be clearly seen descending from various locations, first forming the corona radiata and then converging into the internal capsule. Axons from the caudal/lateral S2 group converged with the main CST just above the cerebral peduncle, and all CSTs descended as a unified tract at that point (Fig. 6A-C). Descending CSTs could be distinguished as they descended into the pyramids and across the medullary decussation. Interestingly, the $3 \mathrm{D}$ reconstruction revealed clear points of collateralization along this path (Fig. $6 A-D$, arrows; Movies 1, 2, 3, and 4). First a cloud-like structure emanated just below the corona radiata and projected into the putamen (Fig. $6 A-D$, purple arrow). Second, extending dorsally from the cerebral peduncles was a tract destined for the pre-tectal and tectal areas (Fig. $6 A-D$, orange arrows; Movie 3). These collaterals were readily detectable as a discrete bundle near their point of departure from the main CST but became more difficult to visualize as they diverged toward distinct targets. By isolating discrete optical slices, a hazy signal was apparent between the mass of CST collaterals and the red nuclei, suggestive of collateral innervation of the red nucleus by CST axons (Movie 4). Finally, near the rostral end of the pyramidal tract, a dense projection extended ventrally, likely corresponding to collateral innervation of the basilar pontine nuclei (Fig. $6 A-D$, green arrows; Movies 1,2). We emphasize that only axons that reached cervical spinal cord were labeled, and thus these collateral tracts clearly indicate the presence of branched projections from definitive CST neurons. To corroborate this finding we examined tracings of individual neurons recently available at the Mouselight Neuron Browser (https://www.janelia.org/project-team/mouselight) and identified six cortical neurons with unequivocal projection to the spinal cord (Economo et al., 2016). Interestingly, all six individual neurons showed branching into pre-tectal areas, five branched into basilar pontine nuclei, and two branched into the putamen (Fig. 6E). Thus, CST axons collateralize extensively in supraspinal nuclei.

3D imaging of the intact brain also clarifies the spatial relationship of supraspinal nuclei and can help draw attention to less-studied populations. For example, 3D imaging of the forebrain reveals not only the CST populations described above, but also supraspinal populations located in the ventral forebrain. These are likely hypothalamic-spinal populations (Liang et al., 2011), appearing here as scattered cells in the lateral and paraventricular hypothalamus (Fig. 7A, B; Movie 5). Similarly, 3D imaging of the red nucleus showed the relative position of the parvocellular and magnocellular populations and the differences in cell morphology between them (Fig. 7C-E; Movie 6). Also apparent in the 3D imaging is a prominent midline group of cells corresponding to the centrally projecting Edinger Westphall nucleus as well as more diffusely distributed cell bodies of the interstitial nucleus of Cajal (Kozicz et al., 2011; Liang et al., 2011; Fig. 7C; Movie 6).

In the cerebellum and brainstem, 3D imaging also helps resolve some of the complexity of the multiple supraspinal populations and their various axon tracts, including spinally projecting reticular neurons, vestibular neurons, and deep cerebellar nuclei (Fig. 7F; Movie 7). Also apparent in the brainstem are the details of trajectories of descending axons. For example, rubrospinal axons enter the upper brainstem as a confined bundle, 

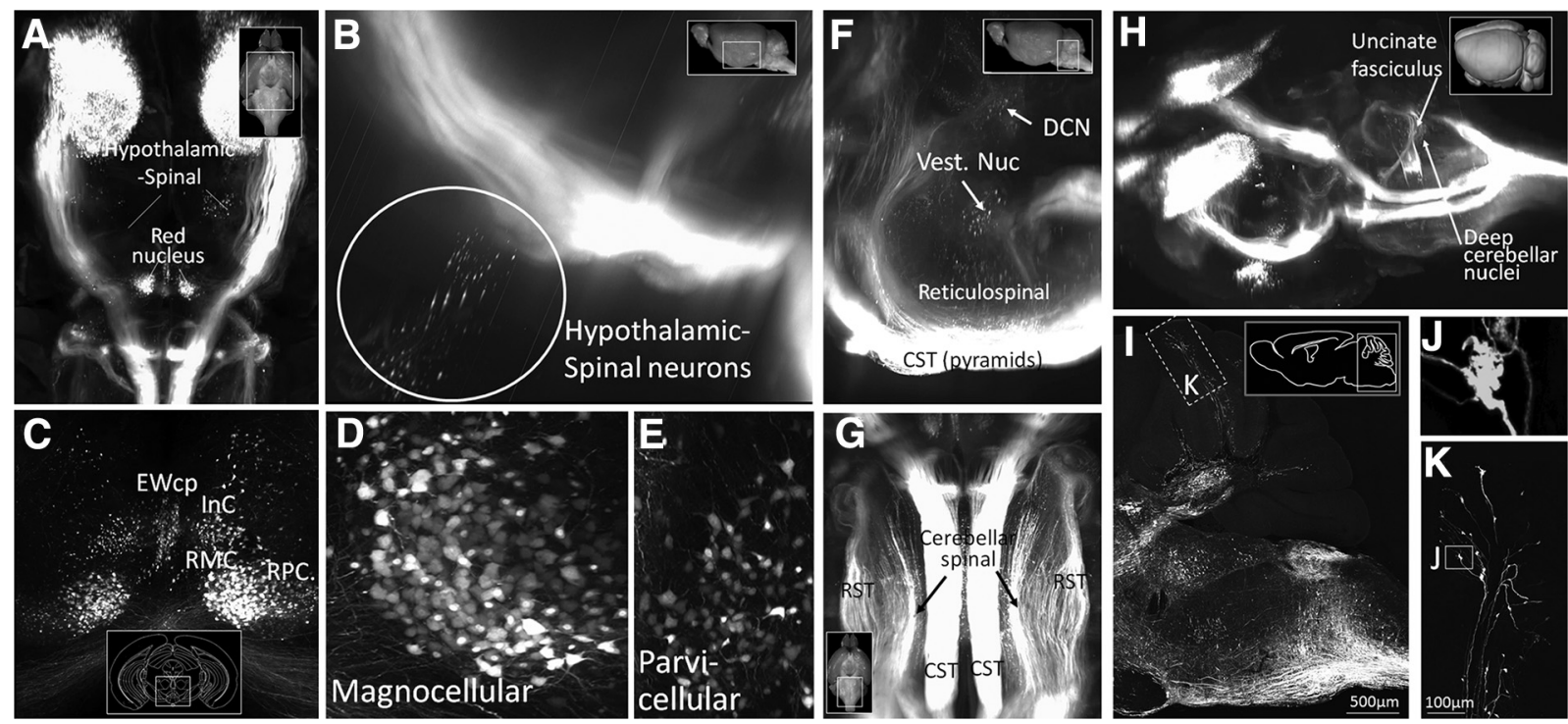

Figure 7. AAV2-Retro and 3D imaging reveal supraspinal populations and axon trajectories. $\boldsymbol{A}-\mathbf{G}$, Adult mice received bilateral cervical injection of AAV2-Retro-tdTomato, and 2 weeks later brains were optically cleared by 3 DISCO and imaged with light-sheet microscopy. $\boldsymbol{A}, \boldsymbol{B}$, Imaging in the diencephalon shows scattered cell bodies likely corresponding to previously described hypothalamic-spinal populations. $\boldsymbol{C}-\boldsymbol{E}$, 3D imaging in the midbrain shows magnocellular (RMC) and parvocellular (PRC) populations of the red nucleus, as well as the centrally projecting component of the Edinger Westphal nucleus (Ewcp) and the interstitial nucleus of Cajal (INC). $\boldsymbol{F}-\boldsymbol{H}, 3 \mathrm{D}$ imaging shows multiple brainstem populations and axon tracts, including the dorsal cerebellar nuclei (DCN), vestibular nuclei, reticulospinal populations, the CST, rubrospinal tract (RST) and cerebellospinal tract. $\boldsymbol{I}-\boldsymbol{K}$, Confocal imaging of sagittal sections of brainstem and cerebellum reveals axons that enter the cerebellar cortex and form large terminals, likely corresponding to mossy fiber terminals of spinocerebellar origin. Videos of cleared tissue are available in Movies 5, 6, and 7.

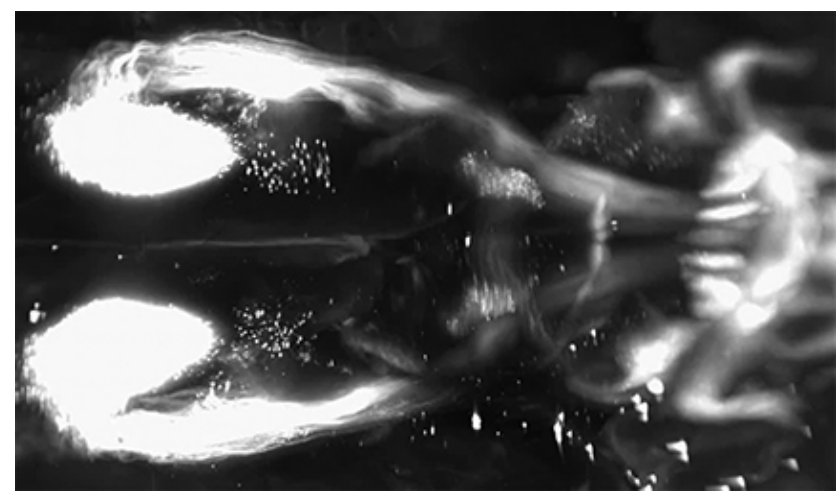

Movie 5. 3D visualization of hypothalamic-spinal projections. Adult mice received bilateral cervical injection of AAV2-Retro-tdTomato. Two weeks later brains were optically cleared by $3 \mathrm{DISCO}$ and imaged with light-sheet microscopy. This video highlights the distribution of hypothalamic-spinal projection neurons located in the ventral forebrain.

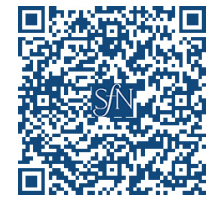

separate in into discrete bundles in the lower pons and upper medulla, then reconverge before entering the spinal cord (Fig. $7 G$; Movie 7). The trajectory of the cerebellar-spinal tract could also be discerned, first extending to the contralateral cerebellum via the uncinate fasciculus (Fig. $7 H$, arrow), then through the superior cerebellar peduncle, and then descending in a position just lateral to the rubrospinal tract. Similar to the rubrospinal tract, the cerebellar-spinal tract broke into discrete bundles as it descended through the brainstem, and then re-aggregated just before entering the spinal cord. (Fig. 7G; Movie 7). In the cerebellar cortex, imaging showed globular structures that initially gave the impression of cell bodies. Closer inspection with highresolution confocal microscopy, however, revealed these to be mossy fiber terminations, likely originating from spinal-cerebellar neurons that were transduced by the spinal injection of AAV2-

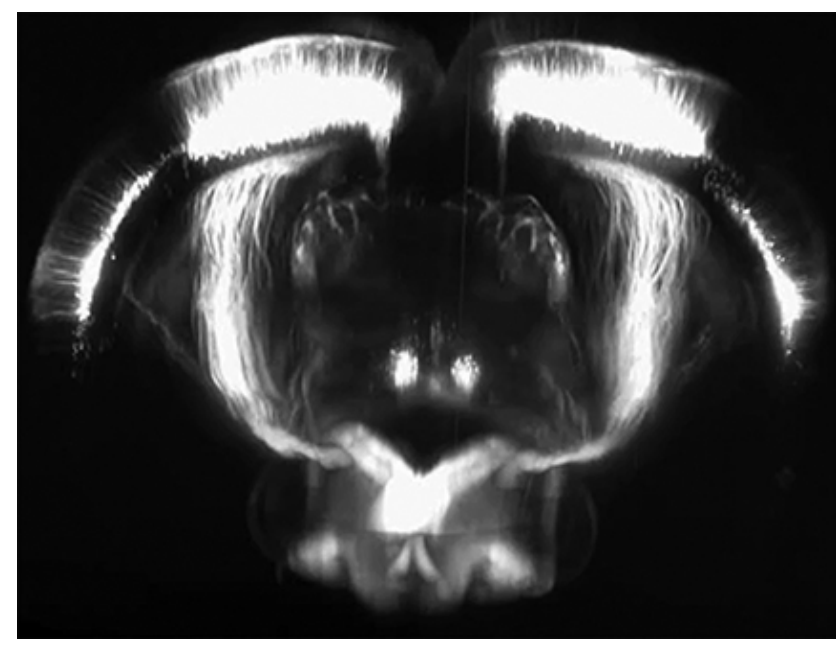

Movie 6. 3D visualization of rubrospinal projections. Adult mice re- $\square$ ceived bilateral cervical injection of AAV2-Retro-tdTomato. Two weeks later brains were optically cleared by $3 \mathrm{DISCO}$ and imaged with lightsheet microscopy. This video illustrates the distribution of parvocellular and magnocellular neurons in the red nucleus, as well as nearby supraspinal populations including EdingerWestphal and the Interstitial Nucleus of Cajal.

Retro-tdTomato (Fig. 7I-K; Sengul et al., 2015). Overall, combining retrograde fluorescent labeling with 3D tissue reconstruction offers a clearer view of the anatomy of supraspinal projections.

Finally, we used combined AAV2-Retro and 3D imaging to visualize the relative positions of neurons that project to cervical versus lumbar spinal cord. AAV2-Retro-EGFP was injected bilaterally to cervical spinal cord of adult mice, and AAV2-RetrotdTomato to lumbar spinal cord. In pilot studies we discovered that unlike cervical injections, lumbar injection of AAV2-Retro required a full 4 weeks to achieve adequate expression level, and 


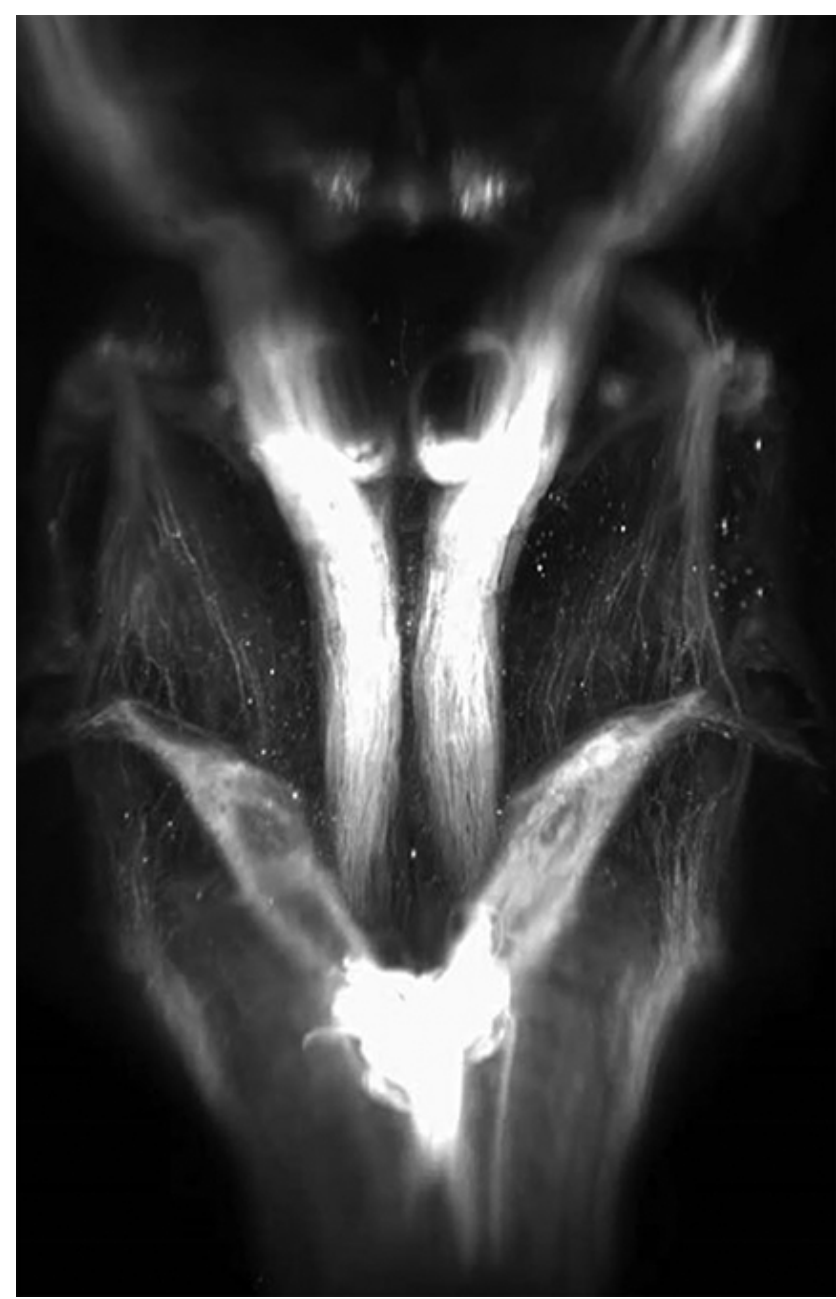

Movie 7. 3D visualization of brainstem-spinal projections. Adult mice received bilateral cervical injection of AAV2-Retro-tdTomato. Two weeks later brains were optically cleared by 3DISCO and imaged with light-sheet microscopy. This video illustrates the diversity of supraspinal projections that originate in the brainstem and the spatial relationships of the descending tracts.

accordingly survival times were increased from 2 weeks to 4 . Consistent with prior work, lumbar-projecting CST neurons (tdTomato + ) were confined to cortex located just caudal to the main population of cervically projecting CST neurons (EGFP+; Kamiyama et al., 2015; Fig. 8A,B; Movie 8). We did not observe any CST neurons that were double-labeled, and no tdTomato + neurons were located in secondary sensory cortex or in the rostral forelimb area, emphasizing the presence of neurons with projections to only lumbar or cervical cord. Similarly, in the red nucleus, cervically-versus lumbar-labeled neurons segregated into two discrete populations, with the lumbar-projecting neurons located lateral to the cervical population with very few double labeled neurons (Flumerfelt and Gwyn, 1974; Liang et al., 2012; Fig. 8C; Movie 8). In the brainstem, tdTomato signal from lumbar spinal cord was widespread in the ventral medulla, likely reflecting reticulospinal projections. In addition, lumbar-derived labeling was apparent in the dorsal aspect of the upper medulla and pons, consistent with prior reports of supraspinal populations in this region including vestibulospinal neurons, the locus ceruleus, and Barrington's nucleus (Liang et al., 2011; Fig. 8D,E; Movie 8). Thus, 3D imaging can also assist in the visualization of the topographical distribution of cell bodies within supraspinal populations that extend axons to different positions along the rostral-caudal axis of the cord. Overall, 3D reconstruction confirms the wide distribution of supraspinal neurons transduced by AAV2-Retro.

\section{Retrograde DREADD silencing of supraspinal populations}

The histological data indicated successful gene delivery to the majority of supraspinal populations with input to cervical spinal cord. To test the functional consequences of silencing the affected neurons we used a DREADD-based strategy, shown previously to effectively silence a variety of supraspinal inputs (Wahl et al., 2014; Siegel et al., 2015; Hilton et al., 2016). We used a Credependent Flex-DREADD construct, in anticipation of future work involving more controlled expression, but in initial experiments deployed the construct in a manner that aimed for widespread and nonspecific DREADD expression. We coinjected AAV2-Retro-Flex-DREADD along with AAV2-Retro-Cre to cervical spinal cord of adult mice, such that all supraspinal neurons that project to cervical spinal cord would have access to both DREADD and the activating CRE. Indeed, mCherry fluorescence confirmed robust DREADD expression in brainstem, red nucleus, and cortical neurons (Fig. 9A).

Starting 2 weeks after AAV injection, we activated DREADD receptors using clozapine. shown by a recent report to function as a DREADD ligand (Gomez et al., 2017). We initially delivered clozapine IP at a concentration of $0.1 \mathrm{mg} / \mathrm{kg}$, a concentration reported to achieve effective DREADD activation (Gomez et al., 2017), and observed animals in open-field locomotion. Within 5 min, animals displayed clear changes in forelimb movement, with a disappearance of plantar placement and the emergence of weight-bearing steps on the curled dorsal surface (Fig. 9B). Within $15 \mathrm{~min}$, weight bearing steps by forelimbs were absent and limbs were pinned beneath the torso. Careful examination showed small, non-effective movements is some animals, and no forelimb movement in others. By $2 \mathrm{~h}$ after injection, most animals returned to weight-bearing steps with non-plantar placement, and by $5 \mathrm{~h}$ showed near-normal locomotion with only occasional non-plantar placement (Fig. 9B, C). To quantify these effects, we established a simple 5-point motor scale from 5 (normal forelimb stepping), 4 (occasional non-plantar steps) 3 (weight bearing steps but completely non-plantar), 2 (no weight bearing steps but occasional large sweeping movements for forward propulsion), 1 (limb predominantly immobile but occasional small movements), and 0 (no discernable movement). We administered a range of clozapine doses, taking advantage of the reversibility of the effects to test a different dose each day. At 0.01 $\mathrm{mg} / \mathrm{kg}$, clozapine produced only a partial loss of plantar placement, and at $0.05 \mathrm{mg} / \mathrm{kg}$ a complete loss of plantar placement but still some weight support. Clozapine at concentrations of 0.1 $\mathrm{mg} / \mathrm{kg}$ and $0.2 \mathrm{mg} / \mathrm{kg}$ produced similar curves, with maximal impairment at $15 \mathrm{~min}$ postinjection $[0.90( \pm 0.34 \mathrm{SEM})$ and 0.95( \pm 0.39 SEM), respectively]. These data establish a dose-response curve for clozapine-mediated DREADD activation, with maximal effects achieved at $0.1 \mathrm{mg} / \mathrm{kg}$. At all doses, effects were maximal at $15 \mathrm{~min}$, partially recovered within an hour, and mostly reversed by $5 \mathrm{~h}$ (Fig. 9C). Finally, we compared these effects to DREADD activation with $\mathrm{CNO}$, an inert compound that has been widely used for DREADD studies, but which was recently reported to be converted to clozapine (Gomez et al., 2017). Consistent with this model, CNO produced paralysis similar to clozapine, but with a delayed and extended time course; effects were maximal at $1 \mathrm{~h}$ and only partially recovered by $5 \mathrm{~h}$ (Fig. $9 \mathrm{C}$ ). Together, these data indi- 

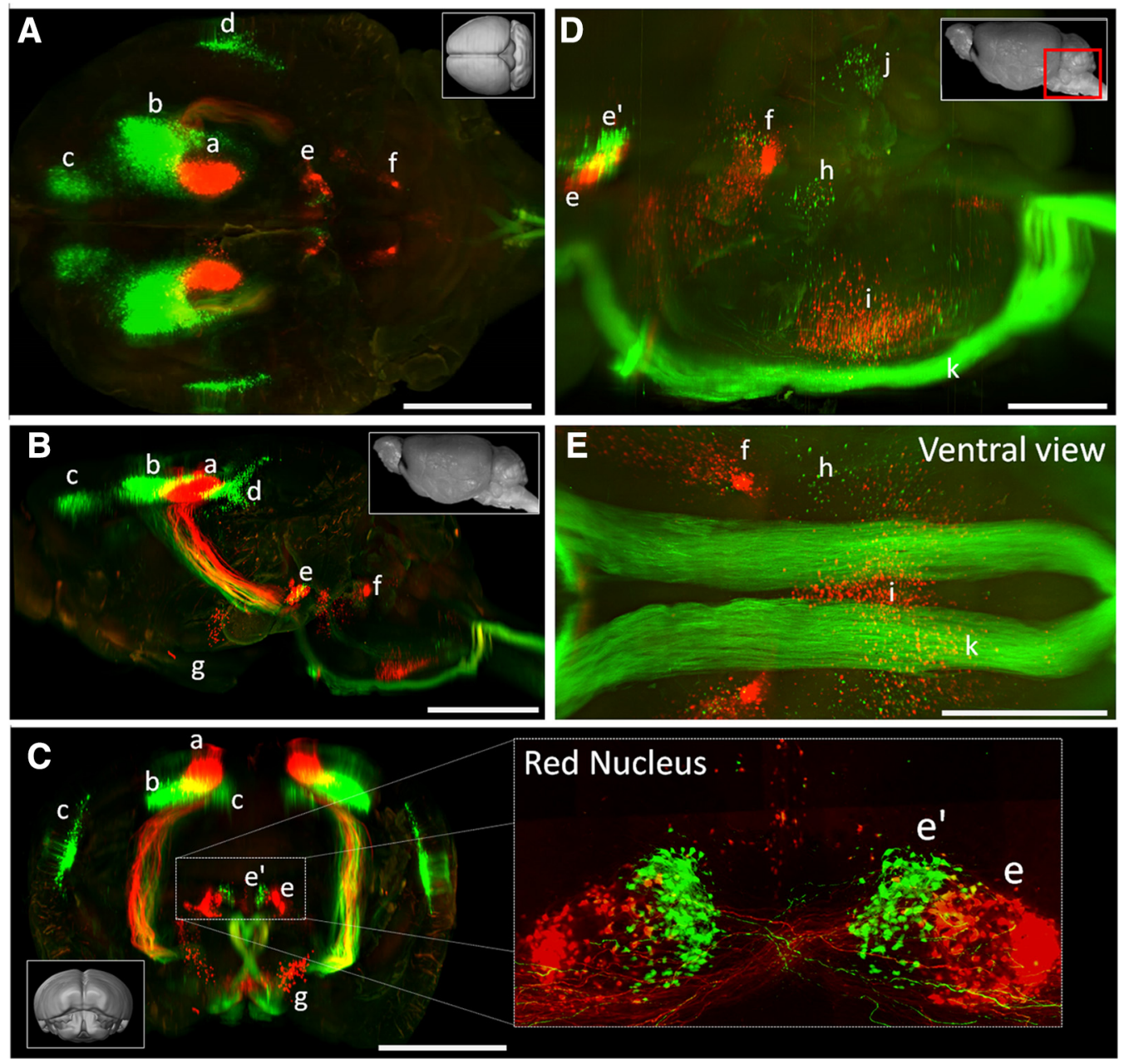

Figure 8. Dual AAV labeling from cervical and lumbar spinal cord shows distinct patterns of innervation. Adult mice received bilateral injections of AAV2-Retro-EGFP to cervical spinal cord, and AAV2-Retro-tdTomato to lumbar spinal cord. Brains were cleared and imaged 4 weeks later. $\boldsymbol{A}-\boldsymbol{C}$, Both CST and rubrospinal cell bodies segregated into distinct lumbar versus cervically-projecting populations. $\boldsymbol{D}, \boldsymbol{E}$, Longer-exposure images of the brainstem reveal distinct populations of lumbar-projecting neurons, including a prominent cluster located in the dorsal pons. $\boldsymbol{a}$, CST, lumbar projecting; $(\boldsymbol{b})$ CST, cervical projecting; (c) CST, rostral forelimb area; (d) CST, secondary sensory; (e) red nucleus, lumbar projecting; ( $\left.\boldsymbol{e}^{\prime}\right)$ red nucleus, cervical projecting; $(\boldsymbol{f})$ mixed dorsal pontine nuclei; $\boldsymbol{g}$ ) hypothalamic-spinal nuclei; $(\boldsymbol{h})$ vestibular nuclei; $(\boldsymbol{i})$ reticular nuclei; $(\boldsymbol{l})$ cerebellospinal nuclei; and $(\boldsymbol{k})$ pyramids of corticospinal tract. Error bars: $\boldsymbol{A}-\boldsymbol{C}, 2 \mathrm{~mm} ; \boldsymbol{D}, \boldsymbol{E}, 1 \mathrm{~mm}$. A video of cleared tissue is available in Movie 8.

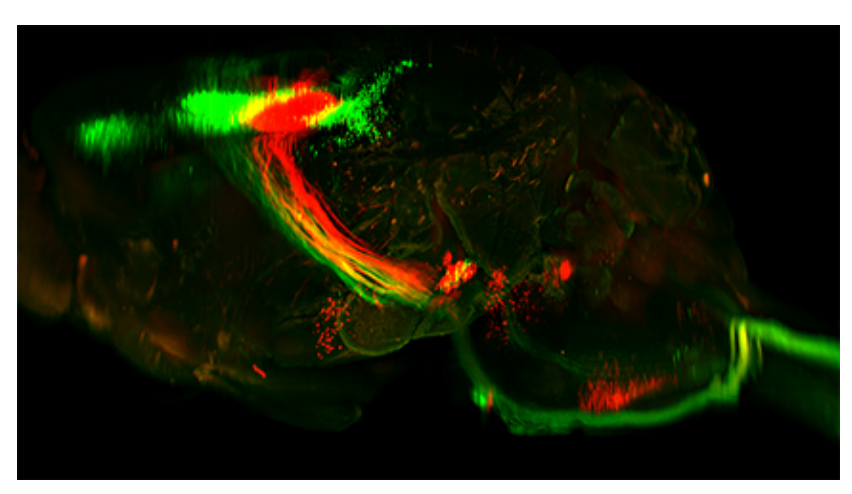

Movie 8. 3D visualization of cervical and lumbar-projecting supraspinal populations. An adult mouse received bilateral injections of AAV2-Retro-EGFP to cervical spinal cord, and AAV2-Retro-tdTomato to lumbar spinal cord. Brains were cleared and imaged four weeks later. This video illustrates CST and rubrospinal populations that project differentially to cervical versus lumbar spinal cord. cate that retrograde delivery of inhibitory DREADDs to cervical spinal cord affects a portion of descending motor input that is sufficient to abolish effective forelimb movement.

To render DREADD expression more specific to CST neurons we injected AAV2-Retro-Flex-Gi-DREADD-mCherry to the cervical spinal cord of CaMKIIa-Cre animals, in which Cre expression is highly enriched in glutamatergic projection neurons in the forebrain (Tsien et al., 1996). In this way, DREADD expression would occur predominantly in corticospinal tract neurons. Indeed, DREADD-injected CaMKIIa-Cre animals showed limited mCherry in brainstem and red nucleus but high expression in layer five of cortex (Fig. 9A). In these animals clozapine injections produced none of the paralysis seen previously and no changes in plantar placement (Fig. 9C), consistent with prior literature showing CST to be dispensable for gross locomotion in mice (Starkey et al., 2005). To test for effects on skilled locomotion we used a modified horizontal ladder task, in which mice were placed atop a wheel with irregularly spaced rungs that rotated at a constant speed (Fig. 9D). The placement of forelimbs was scored by slow-motion video analysis. Clozapine $(0.1 \mathrm{mg} / \mathrm{kg})$ produced a highly repeatable and reversible increase in errors in forelimb placement, which is consistent with selective silencing of CST input $(N=6$, two-way paired $t$ test, $p=$ 
A
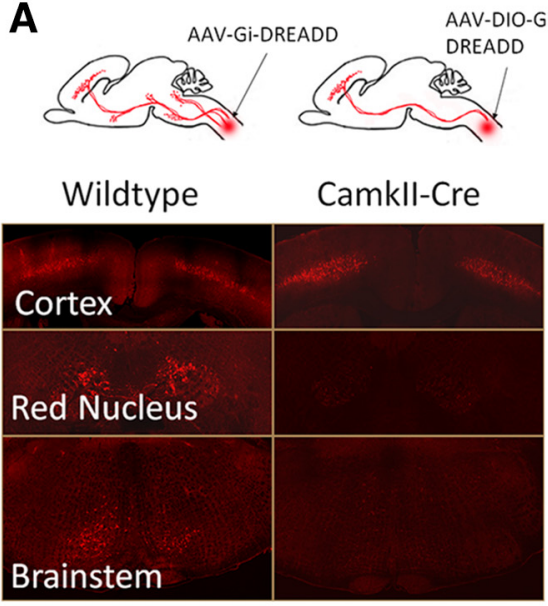

- B

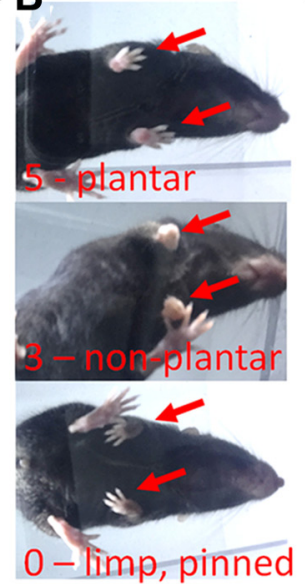

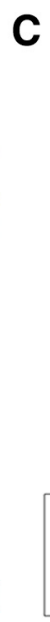

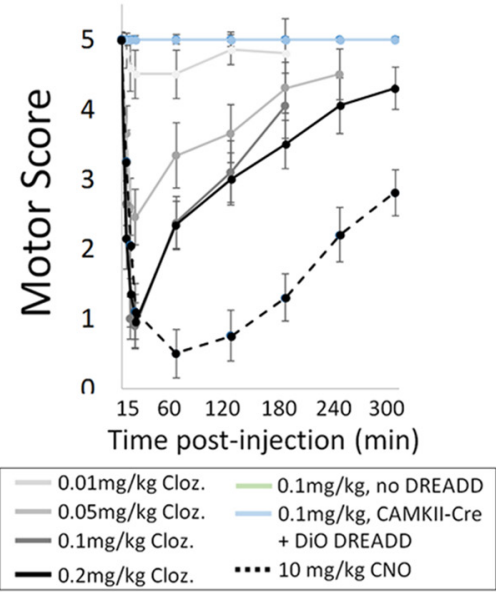

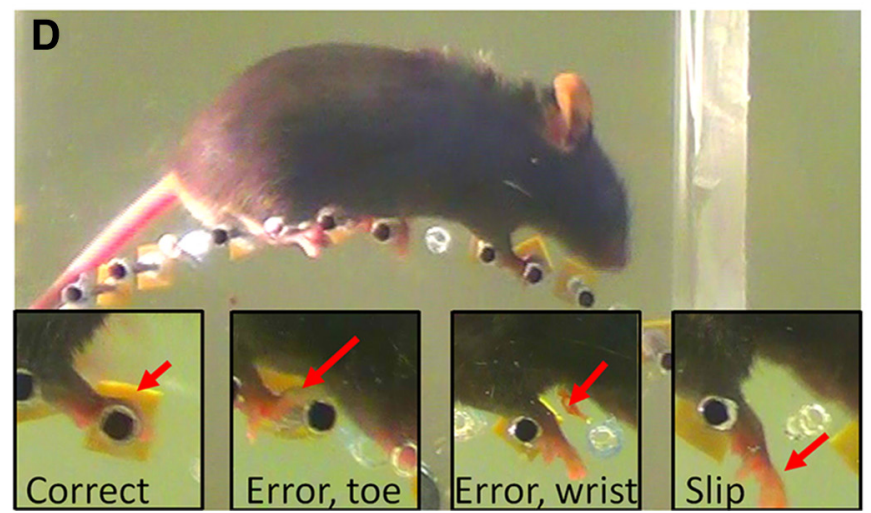

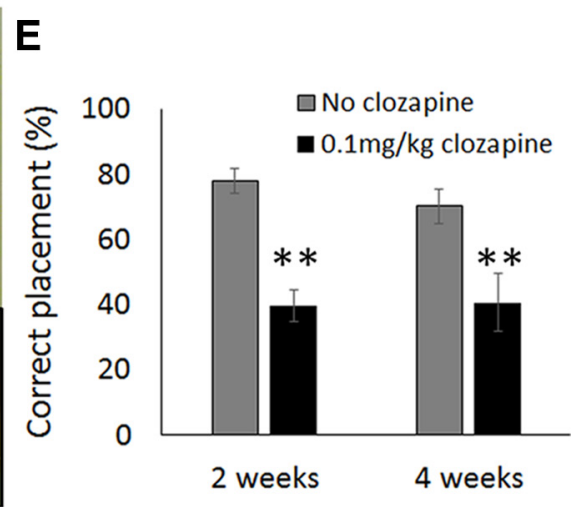

Figure 9. Gi-DREADD-mediated silencing of supraspinal input to cervical spinal cord. $\boldsymbol{A}$, Mixed AAV2-Retro-Flex-Gi-DREADD-mCherry and AAV2-Retro-Cre was injected to the cervical spinal cord of wild-type mice, and Retro-Flex-Gi-DREADD-mCherry alone was injected to the cervical spinal cord of CaMKII-Cre animals. Four weeks later, mCherry signal was readily detectable in the brainstem, red nucleus, and cortex of wild-type animals, but was cortically enriched in Camkll-Cre animals. $\boldsymbol{B}$, Clozapine produce reproducible forelimb deficits in DREADD-injected wild-type animals. $\boldsymbol{C}$, Dose-response and timing curves for clozapine- and CNO-triggered motor deficits. Both ligands triggered reversible forelimb paralysis in Gi-DREADD injected wild-type animals, but not CamkIl-Cre animals or non-injected controls. D, Mice were tested on a horizontal ladder task atop a wheel with irregularly spaced rungs and errors in forelimb placement were scored. $\boldsymbol{E}$, In CaMKIla-Cre animals, clozapine produced significant reduction in correctly targeted steps, consistent with selective silencing of corticospinal tract input to the spinal cord. $\mathrm{N}=5$ wild-type, 4 Camkll-Cre, and 3 non-injected controls. ${ }^{* *} p<0.01$, two-tailed paired $t$ test. Error bars show SEM.

0.0013 at 2 weeks, $p=0.0015$ at 4 weeks; Fig. 9E). Combined, these data highlight the potential of AAV2-Retro-DREADD, combined with transgenic targeting strategies, to probe the functional contribution of individual supraspinal inputs.

\section{Discussion}

This work highlights new approaches for the study and manipulation of supraspinal input to the spinal cord. We showed that AAV2-Retro is broadly effective in transducing most supraspinal populations, including the corticospinal tract, and that transduction is unaffected by acute axon injury. When combined with tissue clearing and 3D reconstruction, retrograde delivery of fluorophores provides interesting insights into the anatomy of descending axon tracts. Finally, retrograde delivery of inhibitory DREADDs is sufficient to block forelimb movements, highlighting the potential for functional dissection of supraspinal inputs to the spinal cord. These data show an important advance in the capacity for retrograde gene delivery to act as a therapeutic tool, as well as to provide anatomical and functional insight into spinal pathways.

\section{AAV2-Retro tropism}

The feature that most distinguishes AAV2-Retro from other AAV serotypes is effective retrograde transduction of CST neurons. Prior work with various AAV serotypes yielded $0-21 \%$ efficiency of retrograde transduction in CST neurons (Jara et al., 2012), and after spinal injury found retrograde transduction in brainstem and rubrospinal neurons but not CST (Klaw et al., 2013). The lentiviral HiRet vector was recently reported to achieve high retrograde transduction rates in CST neurons when injected to early postnatal animals, but these studies left unanswered whether HiRet is similarly effective in adults (Liu et al., 2017; Wang et al., 2017). Here we found that spinal injection of AAV2-Retro in adult animals transduces CST neurons with $>95 \%$ efficiency. Fluorophore expression is quite strong without antibody amplification and emerges within days of injection. AAV2-Retro thus stands out as a powerful new tool for gene delivery to supraspinal populations, including CST.

AAV2-Retro was not as effective in labeling some supraspinal neurons. Most brainstem nuclei were transduced at high rates, but others showed lower efficiencies (Fig. 2). In particular, raphespinal neurons were not transduced, reminiscent of prior observations of low AAV2-Retro transduction in dopaminergic projections (Tervo et al., 2016). Surprisingly, AAV2-Retro was also inefficient in propriospinal neurons. Other AAV serotypes can transduce thousands of propriospinal neurons $>1 \mathrm{~cm}$ from injection sites, (Klaw et al., 2013; Tohyama et al., 2017), yet AAV2-Retro produced sparse transduction just millimeters away. Together, our data indicate that AAV2-Retro uptake is 
highly effective from most projection neurons located in higher brain centers, while other serotypes may be preferable for propriospinal neurons.

Another caveat regards the uptake of AAV2-Retro by axon terminals versus fibers of passage. We found that AAV2-Retro injected to cervical cord transduced CST neurons that innervate the cervical enlargement but did not appear to transduce fibers of passage that innervate lumbar cord. This result hints at preferential access through synaptic terminals. Importantly, however, it remains possible that in different experimental circumstances AAV2-Retro could transduce fibers of passage, for example with injection coordinates that directly target the dorsal CST, different promoters, or more sensitive detection of viral transduction. Even if transduction were purely through synaptic terminals, injured axons are known to sprout and form new synapses above sites of spinal injury (Fouad et al., 2001; Bareyre et al., 2004), potentially creating a new avenue for lumbar-projecting axons to take up cervical AAV2-Retro. It will be important for future work to more completely explore delivery parameters that affect the uptake of AAV2-Retro by injured axons, particularly at more extended times post-injury.

\section{AAV2-Retro and 3D microscopy as a tool for functional neuroanatomy}

Retrograde delivery of cytoplasm-filling fluorophores, combined with tissue clearing and 3D reconstruction, provides new opportunities to clarify aspects of neuroanatomy in the normal, diseased, or injured state. Prior studies with retrograde dyes and 2D imaging have cataloged mouse supraspinal populations (Liang et al., 2011, 2012); the 3D approach now offers a means to place these populations into spatial context while accelerating the task of surveying populations globally. For example, in experimental models of stroke, traumatic brain injury and genetic diseases that involve descending motor pathways, this approach could clarify when and where axonal abnormalities originate, while quantifying in an unbiased way neurons that maintain intact connections (Carron et al., 2016; Sommer et al., 2017). Variable sparing of axons often complicates spinal injury experiments, and thus one application would be to immediately supply AAV2-Retro distal to injury sites, followed by $3 \mathrm{D}$ imaging throughout the brain to rapidly quantify the distribution of virally labeled (spared) axons and neurons.

Injection of AAV2-Retro to cervical spinal cord supplies all branches with cytoplasm-filling fluorophores, helping to clarify points of collateralization along descending axon tracts. Collateral branching has important implications for motor control (e.g., CST axons likely provide a copy of descending motor commands to the cerebellar circuits via pontine collaterals), as well as for regenerative growth (Lorenzana et al., 2015). Recent work has de-emphasized CST collaterals to brain targets (Wang et al., 2017), but the present data reaffirm substantial collateral branching. These branches may stabilize CST axons and perhaps hinder a regenerative response after spinal injury (Lorenzana et al., 2015). In this context, it may be interesting to inject AAV2-Retro to various collateral targets in the brain to compare regenerative growth according the presence of spared collaterals.

One limitation for light-sheet microscopy regards spatial resolution. Light-sheet microscopy detects bundles more effectively than individual thin axons, which may explain why single-cell reconstruction indicates CST collaterals to the medulla that are not detected here (Economo et al., 2016). These collaterals likely exist as individual fibers that are difficult to resolve as they pass near other supraspinal cell bodies and tracts. Transgenic target- ing approaches that remove non-CST fluorescence, combined with traditional sectioning and confocal microscopy, would likely reveal additional CST collaterals in brainstem tissue.

\section{AAV2-Retro as a tool for functional dissection of supraspinal motor control}

Descending motor control signals arise from coordinated activity in widely distributed brain nuclei, and are delivered to the spinal cord in discrete axon tracts (Lemon and Griffiths, 2005; Lemon, 2008; Esposito et al., 2014; Kim et al., 2017). To distinguish their function, a classic method is to monitor motor deficits that follow transection of specific supraspinal tracts. This approach has yielded important insights, for example the partially redundant contributions of corticospinal and rubrospinal tracts to forelimb control (Kanagal and Muir, 2009). A major limitation, however, is the difficulty in specifically ablating tracts by surgical means. Indeed, the CST has been widely studied in part because it is a tractable system for selective ablation in the medullary pyramids. Selective ablation is not always possible, and even in the case of the CST cannot parse functions from discrete cortical subregions. As an alternative, direct injection of toxins or GABA agonists can ablate or silence cell bodies in supraspinal nuclei (Z'Graggen et al., 2000; Esposito et al., 2014). This approach, however, is complicated by issues of specificity, as it is difficult to control the spread of compounds to adjacent and intermingled off-target neurons.

To circumvent these limitations, researchers are increasingly turning to so-called intersectional genetic approaches to silence or kill specific subsets of supraspinal neurons. This strategy pairs injection of retrograde Cre recombinase to the spinal cord with delivery of Cre-dependent constructs to the cell bodies of origin. In this way, only spinally-projecting neurons near the brain injection, and not off-target cells, express the transgene. This approach was used to selectively kill CST neurons in the rostral or caudal forelimb region, revealing differential contributions to reaching and grasping (Wang et al., 2017). Similarly, DREADD expression after stroke or spinal injury has clarified functional contributions of spared CST neurons (Wahl et al., 2014; Hilton et al., 2016).

Our data indicate that AAV2-Retro will be a highly effective addition to these intersectional tools. We found that retrograde delivery of Gi-DREADD to supraspinal neurons causes dramatic and reversible forelimb paralysis, equivalent to the motor effects of a complete spinal transection. Although our expression analysis revealed that some supraspinal neurons escape silencing, their activity was insufficient to achieve movement, highlighting the broad efficacy of AAV2-Retro-DREADD. Importantly, this finding establishes AAV2-Retro as a suitable platform for two complementary types of experiments. Similar to prior work, Credependent AAV2-Retro can be paired with Cre driver lines to probe the necessity of selected supraspinal populations for normal movement. As proof of principle, we confirmed that cortically enriched Gi-DREADD expression causes behavioral deficits that are consistent with selective removal of CST input (Starkey et al., 2005). In addition, it appears that AAV2-Retro now enables complementary experiments to test the sufficiency of individual tracts to evoke movements. By supplying "Cre-off" RETRO-GiDREADD to the spinal cord, while also supplying particular supraspinal regions with Cre (by AAV injection or transgenic means), specific populations could be selectively protected in the context of overall silencing. Indeed, a key question in regeneration research is what percentage of descending neurons, and what type, are needed after injury to carry effective movement. By 
delivering Cre to different supraspinal locations, it will be possible to control the type and percentage of descending axons that escape silencing, and in this way prove their specific contributions. In summary, AAV2-Retro represents an important new tool for the functional dissection of supraspinal control of movement under physiologic and pathological conditions.

\section{References}

Alstermark B, Ogawa J, Isa T (2004) Lack of monosynaptic corticomotoneuronal EPSPs in rats: disynaptic EPSPs mediated via reticulospinal neurons and polysynaptic EPSPs via segmental interneurons. J Neurophysiol 91:1832-1839. CrossRef Medline

Bareyre FM, Kerschensteiner M, Raineteau O, Mettenleiter TC, Weinmann O, Schwab ME (2004) The injured spinal cord spontaneously forms a new intraspinal circuit in adult rats. Nat Neurosci 7:269-277. CrossRef Medline

Blackmore MG, Wang Z, Lerch JK, Motti D, Zhang YP, Shields CB, Lee JK, Goldberg JL, Lemmon VP, Bixby JL (2012) Kruppel-like factor 7 engineered for transcriptional activation promotes axon regeneration in the adult corticospinal tract. Proc Natl Acad Sci U S A 109:7517-7522. CrossRef Medline

Bray ER, Noga M, Thakor K, Wang Y, Lemmon VP, Park KK, Tsoulfas P (2017) 3D visualization of individual regenerating retinal ganglion cell axons reveals surprisingly complex growth paths. eNeuro 4:ENEURO.0093-17.2017. CrossRef Medline

Carron SF, Alwis DS, Rajan R (2016) Traumatic brain injury and neuronal functionality changes in sensory cortex. Front Syst Neurosci 10:47. CrossRef Medline

Castle MJ, Turunen HT, Vandenberghe LH, Wolfe JH (2016) Controlling AAV tropism in the nervous system with natural and engineered capsids. Methods Mol Biol 1382:133-149. CrossRef Medline

Economo MN, Clack NG, Lavis LD, Gerfen CR, Svoboda K, Myers EW, Chandrashekar J (2016) A platform for brain-wide imaging and reconstruction of individual neurons. eLife 5:e10566. CrossRef Medline

Ertürk A, Becker K, Jährling N, Mauch CP, Hojer CD, Egen JG, Hellal F, Bradke F, Sheng M, Dodt HU (2012) Three-dimensional imaging of solvent-cleared organs using 3DISCO. Nat Protoc 7:1983-1995. CrossRef Medline

Esposito MS, Capelli P, Arber S (2014) Brainstem nucleus MdV mediates skilled forelimb motor tasks. Nature 508:351-356. CrossRef Medline

Flumerfelt BA, Gwyn DG (1974) Proceedings: the red nucleus of the rat: its organization and interconnexions. J Anat 118:374,376. Medline

Fouad K, Pedersen V, Schwab ME, Brösamle C (2001) Cervical sprouting of corticospinal fibers after thoracic spinal cord injury accompanies shifts in evoked motor responses. Curr Biol 11:1766-1770. CrossRef Medline

Frampton AR Jr, Goins WF, Nakano K, Burton EA, Glorioso JC (2005) HSV trafficking and development of gene therapy vectors with applications in the nervous system. Gene Ther 12:891-901. CrossRef Medline

Geoffroy CG, Hilton BJ, Tetzlaff W, Zheng B (2016) Evidence for an agedependent decline in axon regeneration in the adult mammalian central nervous system. Cell Rep 15:238-246. CrossRef Medline

Gomez JL, Bonaventura J, Lesniak W, Mathews WB, Sysa-Shah P, Rodriguez LA, Ellis RJ, Richie CT, Harvey BK, Dannals RF, Pomper MG, Bonci A, Michaelides M (2017) Chemogenetics revealed: DREADD occupancy and activation via converted clozapine. Science 357:503-507. CrossRef Medline

Hilton BJ, Anenberg E, Harrison TC, Boyd JD, Murphy TH, Tetzlaff W (2016) Re-establishment of cortical motor output maps and spontaneous functional recovery via spared dorsolaterally projecting corticospinal neurons after dorsal column spinal cord injury in adult mice. J Neurosci 36:4080-4092. CrossRef Medline

Jara JH, Villa SR, Khan NA, Bohn MC, Ozdinler PH (2012) AAV2 mediated retrograde transduction of corticospinal motor neurons reveals initial and selective apical dendrite degeneration in ALS. Neurobiol Dis 47:174183. CrossRef Medline

Kamiyama T, Kameda H, Murabe N, Fukuda S, Yoshioka N, Mizukami H, Ozawa K, Sakurai M (2015) Corticospinal tract development and spinal cord innervation differ between cervical and lumbar targets. J Neurosci 35:1181-1191. CrossRef Medline

Kanagal SG, Muir GD (2009) Task-dependent compensation after pyramidal tract and dorsolateral spinal lesions in rats. Exp Neurol 216:193-206. CrossRef Medline
Kim LH, Sharma S, Sharples SA, Mayr KA, Kwok CHT, Whelan PJ (2017) Integration of descending command systems for the generation of context-specific locomotor behaviors. Front Neurosci 11:581. CrossRef Medline

Klaw MC, Xu C, Tom VJ (2013) Intraspinal AAV injections immediately rostral to a thoracic spinal cord injury site efficiently transduces neurons in spinal cord and brain. Mol Ther Nucleic Acids 2:e108. CrossRef Medline

Kozicz T, Bittencourt JC, May PJ, Reiner A, Gamlin PD, Palkovits M, Horn AK, Toledo CA, Ryabinin AE (2011) The Edinger-Westphal nucleus: a historical, structural, and functional perspective on a dichotomous terminology. J Comp Neurol 519:1413-1434. CrossRef Medline

Kuypers HGJM, Martin GF (1982) Descending pathways to the spinal cord. Progress in Brain Research, Vol 57. Amsterdam: Elsevier.

Lemon RN (2008) Descending pathways in motor control. Annu Rev Neurosci 31:195-218. CrossRef Medline

Lemon RN, Griffiths J (2005) Comparing the function of the corticospinal system in different species: organizational differences for motor specialization? Muscle Nerve 32:261-279. CrossRef Medline

Liang H, Paxinos G, Watson C (2011) Projections from the brain to the spinal cord in the mouse. Brain Struct Funct 215:159-186. CrossRef Medline

Liang H, Paxinos G, Watson C (2012) The red nucleus and the rubrospinal projection in the mouse. Brain Struct Funct 217:221-232. CrossRef Medline

Liu K, Lu Y, Lee JK, Samara R, Willenberg R, Sears-Kraxberger I, Tedeschi A, Park KK, Jin D, Cai B, Xu B, Connolly L, Steward O, Zheng B, He Z (2010) PTEN deletion enhances the regenerative ability of adult corticospinal neurons. Nat Neurosci 13:1075-1081. CrossRef Medline

Liu Y, Wang X, Li W, Zhang Q, Li Y, Zhang Z, Zhu J, Chen B, Williams PR, Zhang Y, Yu B, Gu X, He Z (2017) A sensitized IGF1 treatment restores corticospinal axon-dependent functions. Neuron 95:817-833.e4. CrossRef Medline

Lorenzana AO, Lee JK, Mui M, Chang A, Zheng B (2015) A surviving intact branch stabilizes remaining axon architecture after injury as revealed by in vivo imaging in the mouse spinal cord. Neuron 86:947-954. CrossRef Medline

Nassi JJ, Cepko CL, Born RT, Beier KT (2015) Neuroanatomy goes viral! Front Neuroanat 9:80. CrossRef Medline

Sengul G, Fu Y, Yu Y, Paxinos G (2015) Spinal cord projections to the cerebellum in the mouse. Brain Struct Funct 220:2997-3009. CrossRef Medline

Siegel CS, Fink KL, Strittmatter SM, Cafferty WB (2015) Plasticity of intact rubral projections mediates spontaneous recovery of function after corticospinal tract injury. J Neurosci 35:1443-1457. CrossRef Medline

Soderblom C, Lee DH, Dawood A, Carballosa M, Santamaria AJ, Benavides FD, Jergova S, Grumbles RM, Thomas CK, Park KK, Guest JD, Lemmon VP, Lee JK, Tsoulfas P (2015) 3D imaging of axons in transparent spinal cords from rodents and nonhuman primates. eNeuro 2:ENEURO.000115.2015. CrossRef Medline

Sommer JB, Bach A, Malá H, Strømgaard K, Mogensen J, Pickering DS (2017) In vitro and in vivo effects of a novel dimeric inhibitor of PSD-95 on excitotoxicity and functional recovery after experimental traumatic brain injury. Eur J Neurosci 45:238-248. CrossRef Medline

Starkey ML, Barritt AW, Yip PK, Davies M, Hamers FP, McMahon SB, Bradbury EJ (2005) Assessing behavioural function following a pyramidotomy lesion of the corticospinal tract in adult mice. Exp Neurol 195: 524-539. CrossRef Medline

Tervo DG, Hwang BY, Viswanathan S, Gaj T, Lavzin M, Ritola KD, Lindo S, Michael S, Kuleshova E, Ojala D, Huang CC, Gerfen CR, Schiller J, Dudman JT, Hantman AW, Looger LL, Schaffer DV, Karpova AY (2016) A designer AAV variant permits efficient retrograde access to projection neurons. Neuron 92:372-382. CrossRef Medline

Tohyama T, Kinoshita M, Kobayashi K, Isa K, Watanabe D, Kobayashi K, Liu M, Isa T (2017) Contribution of propriospinal neurons to recovery of hand dexterity after corticospinal tract lesions in monkeys. Proc Natl Acad Sci U S A 114:604-609. CrossRef Medline

Tsien JZ, Chen DF, Gerber D, Tom C, Mercer EH, Anderson DJ, Mayford M, Kandel ER, Tonegawa S (1996) Subregion- and cell type-restricted gene knockout in mouse brain. Cell 87:1317-1326. CrossRef Medline

Wahl AS, Omlor W, Rubio JC, Chen JL, Zheng H, Schröter A, Gullo M, 
Weinmann O, Kobayashi K, Helmchen F, Ommer B, Schwab ME (2014) Neuronal repair. asynchronous therapy restores motor control by rewiring of the rat corticospinal tract after stroke. Science 344:1250-1255. CrossRef Medline

Wang X, Liu Y, Li X, Zhang Z, Yang H, Zhang Y, Williams PR, Alwahab NSA, Kapur K, Yu B, Zhang Y, Chen M, Ding H, Gerfen CR, Wang KH, He Z (2017) Deconstruction of corticospinal circuits for goal-directed motor skills. Cell 171:440-455.e14. CrossRef Medline

Wang Z, Reynolds A, Kirry A, Nienhaus C, Blackmore MG (2015) Overexpression of Sox 11 promotes corticospinal tract regeneration after spinal injury while interfering with functional recovery. J Neurosci 35:31393145. CrossRef Medline
Wu D, Klaw MC, Connors T, Kholodilov N, Burke RE, Côté MP, Tom VJ (2017) Combining constitutively active rheb expression and chondroitinase promotes functional axonal regeneration after cervical spinal cord injury. Mol Ther 25:2715-2726. CrossRef Medline

Z'Graggen WJ, Fouad K, Raineteau O, Metz GA, Schwab ME, Kartje GL (2000) Compensatory sprouting and impulse rerouting after unilateral pyramidal tract lesion in neonatal rats. J Neurosci 20:6561-6569. CrossRef Medline

Zhang YP, Iannotti C, Shields LB, Han Y, Burke DA, Xu XM, Shields CB (2004) Dural closure, cord approximation, and clot removal: enhancement of tissue sparing in a novel laceration spinal cord injury model. J Neurosurg 100:343-352. CrossRef Medline 